\title{
Subtle Impacts of Temperature and Rainfall Patterns on Land Cover Change Overtime and Future Projections in the Mara River Basin, Kenya
}

\author{
Fredrick M. Mngube1", Raphael Kapiyo1, Paul Aboum¹, Douglas Anyona1, Gabriel 0. Dida2,3* \\ ${ }^{1}$ School of Environment and Earth Sciences, Maseno University, Private Bag, Maseno, Kenya \\ ${ }^{2}$ School of Public Health and Community Development, Maseno University, Private Bag, Maseno, Kenya \\ ${ }^{3}$ Department of Health Systems Management and Public Health, Technical University of Kenya, Nairobi, Kenya \\ Email: ^fredmhina@yahoo.com, ${ }^{\star}$ gdidah@gmail.com
}

How to cite this paper: Mngube, F.M., Kapiyo, R., Aboum, P., Anyona, D. and Dida, G.O. (2020) Subtle Impacts of Temperature and Rainfall Patterns on Land Cover Change Overtime and Future Projections in the Mara River Basin, Kenya. Open Journal of Soil Science, 10, 327-358.

https://doi.org/10.4236/ojss.2020.109018

Received: July 17, 2020

Accepted: September 7, 2020

Published: September 10, 2020

Copyright $\odot 2020$ by author(s) and Scientific Research Publishing Inc. This work is licensed under the Creative Commons Attribution International License (CC BY 4.0).

http://creativecommons.org/licenses/by/4.0/

(c) (i) Open Access

\begin{abstract}
The interactive and cumulative effect of temperature and rainfall on land cover change is a priority at global, regional and local scale. This study examined changes in six land cover categories (forestland, grasslands, shrub land, bare land, built-up areas and agricultural lands) in four sub-catchments (Amala, Nyangores, Talek and Sand River), of the Mara River basin over a 30-year period (1987-2017) and made predictions of future land cover change patterns. Landsat Imageries of $90 \mathrm{~m}$ resolution were retrieved and analyzed using ArcGIS 10.0 software. Relationship between NDVI, temperature and precipitation was determined using Pearson's correlation coefficient, while Markov chains analyses were performed on different land cover categories to project future trends. Results showed low to moderate $\left(R^{2}=\right.$ 0.002 to 0.6 ) trends of change in NDVI of different land cover categories across all sub-catchments. The greatest change $\left(R^{2} 0.34\right.$ to 0.5$)$ was recorded in bare land in three of the four sub-catchments studied. Precipitation showed a strong positive correlation with built-up areas, forestlands, croplands, bare land, grasslands and shrub lands, while temperature correlated strongly but negatively with the same land cover categories. The change detection matrix projected significant but varying changes in land cover categories across the four sub-catchments by 2027 . This study underscores the impact of changing climatic factors on various land cover categories in the Mara River basin sub-catchments, with different land cover categories exhibiting strong positive sensitivity to high precipitation and low temperature and vice-versa.
\end{abstract}




\section{Keywords}

Land Cover, Change Detection, Temperature, Rainfall, Sub-Catchments, Mara River, Kenya

\section{Introduction}

Changes in land cover (LC) pattern globally reflect the interaction between human activities and the natural environment [1]. Climatic and land cover changes are increasingly becoming important components of sustainability especially for aquatic ecosystems [2] [3]. Due to anthropogenic activities, the Earth surface is under continuous alteration that impacts heavily on the natural environment [4]. Studies have linked changes in land cover to increased anthropogenic activities [5]. While the role of climate on land cover change has been extensively researched and discussed at the global and regional scales, knowledge of their impact at the local sub-catchment scale is limited, disjointed and anecdotal to draw any meaningful conclusions.

Studies show that the impacts of temperature and precipitation on land cover change are complex [6] and therefore require area specific studies to understand their correlation. This is because global analysis of the relationship between NDVI, precipitation and land surface temperature gives different views. While some researchers have not found any significant correlation [7], others have reported negative or positive relationships between climatic factors and land cover categories [8] [9]. A study in the northeast China by Luo et al. [10] established presence of a strong relationship between NDVI, precipitation and temperature for different vegetation types. The effect of temperature on NDVI was more obvious than that of precipitation in that particular study [10].

Zhang et al. [11] also reported the existence of a positive correlation between NDVI and temperature but pointed out that the effect of precipitation on NDVI was not as significant. Additionally, Zhang et al. [11] established that bushland NDVI correlated more strongly with precipitation than NDVI of other vegetation [11]. Based on these observations, it is apparent that global and regional responses to climate change show wide variation [12]. Therefore, there is a need to undertake studies that quantitatively measure the effect of changes in climatic factors on land cover change at the local level.

Given its many advantages, NDVI is best suited to monitor local or global vegetation changes resulting from a changing climate [13] [14] [15].

Normalized Difference Vegetation Index has been widely used for studying climatic effects on vegetation productivity since the 1980s, though results vary by complexity of vegetation characteristics and region [16] [17]. It is predicted that by 2050 , temperature and precipitation are likely to show decreasing and increasing signals, respectively, across the East African region [18]. However, the magnitude of change is likely to vary by region and location. Predicting land 
cover change is therefore important in understanding and highlighting potential modifications and alterations that are likely to happen over landscapes in the near future. Such projections are useful to land use planners, resource managers, and conservation practitioners in their attempt to manage and mitigate impacts [19]. Prediction of LC change has been used in different applications, such as urban planning [20]; selection of conservation priority areas and setting alternative conservation measures [21] studying dynamics of shifting cultivation [22] and in simulation of rangeland dynamics under different climate change scenarios [23]. A solid understanding of the trends in land cover change at different time scales (past, present and future) at the local scale is therefore critical for decision making and policy formulation.

A review of the most commonly used approaches to modeling and land use change prediction can be found in a study by Agrawal et al. [24]. Markov chain analysis has been extensively used to study dynamics of land use change at different scales [25]. It is a simple method for modeling land use change especially at large scales [26]. The stationary transitions assumed by the Markov chain models make it suitable for short-term projections [27]. However, given its' shortcomings, Markov chain analysis is often integrated with other empirical models [28]. The Markov-CA approach used in the current study is considered a spatial transition model as it combines the stochastic spatial Markov techniques with the stochastic spatial cellular automata method [29]. It has the advantage of predicting two-way transitions among the available LC classes, in contrast to the Geomod technique that only predicts one-way loss/gain from one class to another [30]. Lu et al. [20] noted that transition-based models that integrate spatial Markov model with spatial cellular automata model outperformed regression based models in predicting land use change.

Mara River basin of Kenya which supports the great wildlife migration has witnessed remarkable expansion, growth and development since 1980s, just like many other river sub-catchments in Kenya. Coupled with current innuendo of climate change, anthropogenic activities have resulted in increased land cover modification and alterations over time. However, there is limited information available on the extent to which climate change has impacted the past and present land cover types as well as future impacts in the four sub-catchments of Mara River basin, Kenya. This study is a comprehensive attempt to evaluate the past, present and predict future land cover changes resulting from climate change so as to provide policy and decision makers with a basic tool for future planning.

\section{Materials and Methods}

\subsection{Study Area}

The Mara River Basin (Figure 1), situated between latitudes $0^{\circ} 21^{\prime} \mathrm{S}$ and $1^{\circ} 54^{\prime} \mathrm{S}$ and longitudes $33^{\circ} 42^{\prime} \mathrm{E}$ and $35^{\circ} 54^{\prime} \mathrm{E}$ is shared between Kenya $65 \%\left(16,320 \mathrm{~km}^{2}\right)$ and Tanzania $35 \%\left(8030 \mathrm{~km}^{2}\right)$ [31]. On the Kenya side, the Mara basin is bound 




Figure 1. Map of the study area.

to the west by the Soit Ololo Escarpment, to the east by the Loita and Sannia Plains and to the southwest by Mau forest reserve. Both large and small scale agriculture is practiced in the basin alongside other anthropogenic activities. Livestock farming is also an important economic activity within the basin. The main livestock reared is the zebu cattle, small East African goats and red Maasai hair sheep. Dairy farming is limited to high potential areas where the mean annual rainfall is $1000 \mathrm{~mm}$. Grasslands and shrub lands are the dominant vegetation in the Maasai Mara National Reserve. The major tributaries of the Upper Mara Basin are Amala, Nyangores, Talek and Sand River. Indiscriminate deforestation along the Mau Hills; the source of Mara River, is considered the main cause of high flow fluctuations of the Mara River. There is a constant mean monthly maximum temperature of $28^{\circ} \mathrm{C}$ throughout the year, with a mean minimum temperature range of between $16^{\circ} \mathrm{C}$ in the hot months (October to March) and $13^{\circ} \mathrm{C}$ in the cooler months (May to August).

\subsection{Data Acquisition, Sources and Processing}

Land cover data was derived from Landsat images that were downloaded from the earth explorer United States Geology Survey (USGS) website. The maximum likelihood method was used for the land cover classification in Earth Resource Development Assessment System (ERDAS) Imagine 2013 software. Images were obtained from the three scenes of Landsat $4 \& 5$, Landsat 7 and Landsat 8 , 
Path/Row: 169/060, 169/61 and 170/61, respectively. The Digital Elevation Model (DEM) of $90 \mathrm{~m}$ resolution was obtained from the NASA Shuttle Radar Topography Mission (SRTM) and delineated/processed using ArcMap software.

Land cover classification covering the four sub-basins (Amala, Nyangores, Talek and Sand River) was processed using ERDAS Imagine 2013. The year 1987 was taken as base year of study while Landsat imageries were used due to their suitability for vegetation cover analysis especially vegetation discrimination, measurement of chlorophyll absorption and vegetation type and biomass content analysis. The Landsat imageries used were for October (driest month) for the 1987, 1997, 2007 and 2017 intervals, believed to be reasonable to give substantial changes in land cover. The geo-referencing and image thinning was applied with a resolution of $90 \mathrm{~m}$ using a factor of two to modify its properties and resolution. Image thinning was carried out through contract; which generalizes an image by reducing the number of rows and columns while simultaneously decreasing the cell resolution. With pixel thinning, every $n^{\text {th }}$ pixel was kept while the remaining discarded. Based on the priori knowledge of the study area for over 30 years and a brief reconnaissance survey, a classification scheme was developed for the study area. The classification scheme developed gives a rather broad classification where the land cover was identified by a single digit as shown in Table 1.

\subsubsection{Normalized Difference Vegetation Indices (NDVI)}

The NDVI images for the month of October between 1987 and 2017 were utilized to obtain specific NDVI values. The random points were generated for specific land cover category and the multi values extracted using geo-statistical tool. Land cover was overlaid on top of the NDVI image in order to extract random points of a specific land cover i.e. forestland, bare land, grassland, cropland, and built up areas. The generated points were later extracted through a process of geo-statistical analysis by multi values regression function. The random points were separately obtained through computed averages for respective monthly NDVI values in order to get the mean monthly NDVI for specific land category.

Table 1. Land cover classification scheme.

\begin{tabular}{cc}
\hline & Land Use/Land Cover Categories \\
\hline 1 & Crop land \\
2 & Bare land $^{*}$ \\
4 & Built-up areas \\
5 & Forest land \\
6 & Shrub land \\
\hline
\end{tabular}

*The definition of bare land as used in this study denotes land without shrub, sandy areas, dry grasses, rocky areas and other human induced barren lands. 


\subsubsection{Transition Probability Matrix}

The transition probability matrix records the probability that each land cover category will change to another or remain in the same category. For the 6 by 6 matrix table, the rows represent land cover categories and the column represents corresponding NDVI values. Although this matrix can be used as a direct input for specification of the prior probabilities in maximum likelihood classification of the remotely sensed imagery, it was used in predicting land cover change by 2027.

\subsubsection{Temperature and Precipitation Data Acquisition and Analysis}

Temperature and rainfall data sets were obtained from Giovanni website. The data obtained were of high resolution $\left(0.1^{\circ}\right.$ latitude $\times 0.1^{\circ}$ longitude) daily gridded sets. Averages were calculated to obtain monthly and annual mean temperature and precipitation for the period between 1987 and 2017 (October). The Normalized Difference Vegetation Index technique was used to extract the various features presented in satellite imagery. Vegetation indices allowed the delineation of vegetation distribution and soil, based on the characteristic reflectance patterns of green vegetation. NDVI of the four sub-basins were generated following their different land cover categories in the study area. This yielded different NDVI values depending on vegetation healthiness and extend.

The NDVI images for the month of October (driest month) from the years 1987 to 2017 were utilized to obtain specific NDVI values. Random points were generated for specific land use category and the multi values extracted using geo-statistical tool. Land cover was overlaid on NDVI images to extract random points of specific land cover types i.e. forest, bare land, grassland, shrub land, cropland, and built up areas. The generated points were later extracted through a process of geo-statistical analysis by multi values regression function. The process is as shown in Figure 2.

\subsubsection{Impacts of Temperature and Precipitation on Different Land Cover Categories}

Kriging methods were employed in ArcGIS to produce monthly and annual

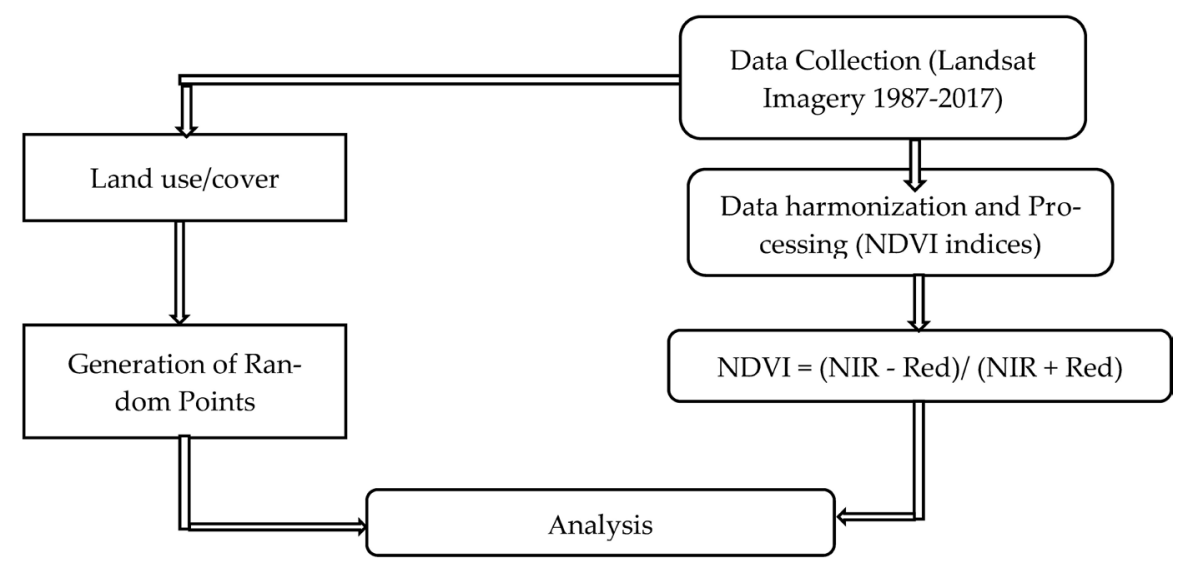

Figure 2. NDVI process. 
precipitation and temperature gridded maps for each sub-basin. The average values of NDVI, temperature and precipitation associated with a particular vegetation type were calculated from the averages of all Pearson's correlations (P) between monthly variables in MS Excel and P-values used to determine significance levels. Considering the lagged response of NDVI to temperature and precipitation, the correlation analyses were also carried out between each seasonal NDVI and the previous season's temperature and precipitation for the month of October.

\subsubsection{Determination of the Accuracy of Land Cover Maps}

The overall accuracy of the land cover maps for 1987, 1997 and 2017 was determined and the Validation Kappa indices for each map were: 0.901 for Amala, 0.898 for Nyangores, 0.938 for sand and 0.963 for Talek. The land cover maps in the study area from 1987 to 2017 are shown in the results section. A confusion matrix was applied in the present study to check the accuracy of the results. The overall accuracy and Kappa concordance coefficient of agreements (Kappa coefficient) in each year were calculated, and finally the overall accuracy of all classification results obtained as an average value. The equation of Kappa coefficient is as shown below [32]. If $p_{a}=$ the proportion of observations in agreement and $p_{\varepsilon}=$ the proportion in agreement due to chance, then Cohen's kappa is:

$$
k=\frac{p_{a}-p_{s}}{1-p_{s}}
$$

Alternatively

$$
k=\frac{n_{a}-n_{s}}{n-n_{s}}
$$

where $n=$ number of subjects, $n_{a}=$ number of agreements and $n_{s}=$ number of agreements due to chance.

\subsubsection{Forecast}

Comparison of the land cover statistics assisted in identifying the percentage change, trend and rate of change between 1987 and 2017. The first task was to develop a table showing the area in hectares and the percentage change for each of the 10 years (Decadal; 1987, 1997 and 2017) measured against each land cover category. Percentage change to determine the trend of change was then calculated by dividing observed change by sum of changes then multiplied by 100 .

$$
\text { (Trend) Percentage change }=\frac{\text { Observed change } \times 100}{\text { Sum of change }}
$$

In obtaining annual rate of change, the percentage change was divided by 100 and multiplied by the number of study years 1987-1997 (10 years) 1987-2007 (20 years).

\subsubsection{Markov Chain Analysis}

Markov chains were used to obtain the percentage and probability for each cat- 
egory of land cover converted. Using the Markov model, the distribution of each land cover category was projected based on the transition probability $p_{i j}$ between two land cover categories ( $i$ and $j)$. $P_{i j}$ was determined over a specific period, from time $t$ to time $t+1$, as follows:

$$
P_{i j}=P\left[x_{n+1}=j \mid x_{n}=i\right] \ldots[t t+1]
$$

Let $P=P_{i j}$ denote the (possibly infinite) transition matrix of the one-step transition probabilities

where:

$P=$ the Markov transition matrix $P$

$i, j=$ the land type of the first and second time period

$P_{i j}=$ the probability from land type $i$ to land type $j$

$t t+1=$ time

The estimate of Markov chain is the relative frequency of transitions observed over the entire time period. The result of the estimation was used for prediction. In practice, based on the map algebra principle, the class of land type utilizes the equation below to calculate the transfer map of land cover change under the ERDAS Modeler module.

$$
\begin{gathered}
C_{i j}=A_{i j}^{t} X^{10}+A_{i j}^{t+1} \\
C_{I J}=A_{i j}^{t} X^{10}+A_{i j}^{t+1}
\end{gathered}
$$

where:

$A_{i j}^{t}, A_{i j}^{t+1}=$ the land use map of the first and second time period, respectively

$t, t+1=$ the first and the second time period

$i, j=$ the land type of the first and second time period

$C_{i j}=$ the class of land type $i$ to land type $j$

To establish the trajectory of land cover change, numerous sample points were selected over the study area and land cover category at every period recorded using GIS functions in ArcInfo Tool box. Predictions of future land cover change using a Markov-CA model was done in the following steps:

1) Applying the Markov chain analysis to the 2007 and 2017 developed maps for calculating transition matrices;

2) Calculating land cover change transition potential maps; and

3) Predicting the land cover change for 2027 by using Markov CA model to develop transition matrices and then developing transition potential maps for all four sub-basins.

To understand decadal land cover dynamics, the periods between 1987-1997; 1997-2007 and 2007-2017, were used to produce land cover dynamics for each sub-basin. These dynamics helped to highlight changes to different land cover categories (what gained and lost and from which land cover category to another). The 2007 and 2017 land cover maps were then used to predict the changes likely to occur in land cover by 2027 for each sub-basin. 


\section{Results}

\subsection{Land Cover Changes between 1987 and 2017 across the Four Mara River Sub-Catchments}

Overall, a considerable reduction in the spatial expansion of LC was observed between 1987 and 1997 compared to the period between 2007 and 2017 across all the four Mara River sub-catchments.

\subsubsection{Nyangores Sub-Catchment}

The findings presented in Table 2 show the static area of each NDVI of land cover category for each study year in the Nyangores sub-basin. Bare lands $(-0.06)$ and built-up area $(-0.06)$ occupied the least class with just $-15.4 \%$ and $-15.0 \%$ respectively, of the total classes in 1987 compared to 2017 (Figure 3). A similar trend was observed in areas under forest cover, crop land and grassland. Generally, when mean total annual precipitation was high and maximum mean annual temperature was low, the NDVI of all land cover categories was highest (see year 2007). On the contrary, when maximum mean annual temperature was high and mean total annual precipitation was low, NDVI of all land cover categories was lowest (see year 1997). A decrease of $-478.6 \mathrm{~mm}$ in precipitation, from $1171 \mathrm{~mm}$ to $692 \mathrm{~mm}$-translating to $-40.87 \%$ was observed between 1987 and 2017 in Nyangores sub-catchment. However, maximum mean annual temperature increased from $22.54^{\circ} \mathrm{C}$ to $26.96^{\circ} \mathrm{C}$-a difference of $2.23^{\circ} \mathrm{C}$, translating to $9.89 \%$ increase.

Results further showed trends in change in NDVI of land cover categories, particularly in dry season (October) from low $\left(\mathrm{R}^{2}=0.002\right.$ to 0.17$)$ to moderate (0.3 to 0.5$)$ variation. Variation in mean crop land cover category NDVI was $\mathrm{R}^{2}$ $=0.002$, shrub land NDVI was $\mathrm{R}^{2}=0.03$, forest land cover NDVI was $\mathrm{R}^{2}=0.09$; grassland was $\mathrm{R}^{2}=0.17$, while built up area was $\mathrm{R}^{2}=0.30$. The greatest change was observed in bare land $\left(\mathrm{R}^{2}=0.50\right)$. Temperature variation trend also showed relative low change in mean $\left(\mathrm{R}^{2}=0.32\right)$ while rainfall showed greater change in mean $\left(\mathrm{R}^{2}=0.62\right)$. The Pearson correlation coefficient between annual mean precipitation and built-up areas, forest cover, crop land, bare land, grasslands and

Table 2. NDVI per land cover category in Nyangores sub-catchment (1987-2017).

\begin{tabular}{|c|c|c|c|c|c|c|c|c|c|c|c|c|}
\hline YEAR & $\begin{array}{c}\text { NDVI } \\
1987\end{array}$ & $\begin{array}{c}\text { NDVI } \\
1997\end{array}$ & $\begin{array}{l}\text { NDVI } \\
\text { change } \\
1987 \text { to } \\
1997\end{array}$ & $\begin{array}{c}\text { NDVI \% } \\
\text { change } \\
1987 \text { to } \\
1997\end{array}$ & $\begin{array}{c}\text { NDVI } \\
2007\end{array}$ & $\begin{array}{l}\text { NDVI } \\
\text { Change } \\
1997 \text { to } \\
2007\end{array}$ & $\begin{array}{c}\text { NDVI \% } \\
\text { change } \\
1997 \text { to } \\
2007\end{array}$ & $\begin{array}{c}\text { NDVI } \\
2017\end{array}$ & $\begin{array}{l}\text { NDVI } \\
\text { Change } \\
2007 \text { to } \\
2017\end{array}$ & $\begin{array}{l}\text { NDVI \% } \\
\text { change } \\
2007 \text { to } \\
2017\end{array}$ & $\begin{array}{l}\text { NDVI } \\
\text { Change } \\
1987 \text { to } \\
2017\end{array}$ & $\begin{array}{c}\text { NDVI \% } \\
\text { change } \\
1987 \text { to } \\
2017\end{array}$ \\
\hline BUILT & 0.4 & 0.34 & -0.06 & -15.0 & 0.44 & 0.1 & 29.41 & 0.25 & -0.19 & -43.18 & -0.15 & -37.5 \\
\hline FOREST & 0.6 & 0.53 & -0.07 & -11.7 & 0.75 & 0.22 & 41.51 & 0.42 & -0.33 & -44.00 & -0.18 & -30.0 \\
\hline CROP & 0.46 & 0.35 & -0.11 & -23.9 & 0.6 & 0.25 & 71.43 & 0.39 & -0.21 & -35.00 & -0.07 & -15.2 \\
\hline BARE & 0.39 & 0.33 & -0.06 & -15.4 & 0.4 & 0.07 & 21.21 & 0.19 & -0.21 & -52.50 & -0.2 & -51.3 \\
\hline GRASS & 0.56 & 0.42 & -0.14 & -25.0 & 0.59 & 0.17 & 40.48 & 0.4 & -0.19 & -32.20 & -0.16 & -28.6 \\
\hline SHRUB & 0.48 & 0.41 & -0.07 & -14.6 & 0.57 & 0.16 & 39.02 & 0.39 & -0.18 & -31.58 & -0.09 & -18.8 \\
\hline
\end{tabular}






Figure 3. October land cover categories NDVI from 1987 to 2017 in Nyangores sub-catchment.

shrub land NDVI was $0.94 ; 0.82,0.52,0.99,0.80$, and 0.71 , respectively (Table 3 ).

Findings further showed that precipitation affected NDVI positively. Regarding the Pearson's correlation coefficient between maximum mean temperature and land cover (built-up areas, forest cover, crop land, bare land, grasslands and shrub land) NDVI were $-0.99,-0.96,-0.77,-0.98,-0.93$ and -0.9044 , respectively. In Nyangores, maximum temperature affected NDVI negatively. Temperature showed less variation $\left(\mathrm{R}^{2}=0.32\right)$ while precipitation showed greater variation $\left(R^{2}=0.62\right)$. Due to the change in maximum mean annual temperature $\left(R^{2}=\right.$ $0.32)$ and mean total annual precipitation $\left(\mathrm{R}^{2}=0.62\right)$, land cover categories have been affected differently (Table 3, Table 4 and Figure 3). Generally, land cover categories appeared more sensitive to precipitation than temperature in Nyangores sub-catchments.

\subsubsection{Amala Sub-Catchment}

In the Amala sub-basin, changes were observed in different land cover categories i.e. cropland $\left(\mathrm{R}^{2}=0.06\right)$; built up with $\left(\mathrm{R}^{2}=0.12\right)$; forest land cover $\left(\mathrm{R}^{2}=0.14\right)$; shrub of $\left(\mathrm{R}^{2}=0.30\right)$; grass with $\left(\mathrm{R}^{2}=0.31\right)$ in the month of October. The greatest change was observed in bare land of $\mathrm{R}^{2}=0.34$. Temperature also showed relative low change in mean $\left(R^{2}=0.32\right)$ while rainfall showed a high change in mean $\left(R^{2}\right.$ 
Table 3. Trend of rainfall and temperature in Nyangores sub-catchment.

\begin{tabular}{|c|c|c|c|c|c|c|c|c|}
\hline YEAR & 1987 & 1997 & $\begin{array}{l}\text { Change btw } \\
1987 \& 1997\end{array}$ & 2007 & $\begin{array}{l}\text { Change btw } \\
1997 \& 2007\end{array}$ & 2017 & $\begin{array}{l}\text { Change btw } \\
2007 \& 2017\end{array}$ & $\begin{array}{l}\text { Change btw } \\
1987 \& 2017\end{array}$ \\
\hline PPT annual mean & 1171 & 1038.6 & -132.4 & 1139.6 & 101 & 692.4 & -447.2 & -478.6 \\
\hline $\begin{array}{l}\text { TEMP max annual } \\
\text { mean }\end{array}$ & 22.54 & 23.48 & 0.94 & 22.03 & -1.45 & 24.77 & 2.74 & 2.23 \\
\hline
\end{tabular}

Table 4. Person correlation between temperature, precipitation and NDVI of different land cover categories between 1987-2017 in Nyangores sub-catchment.

\begin{tabular}{cccccccccc}
\hline Year & $\begin{array}{c}\text { PPT annual } \\
\text { mean }\end{array}$ & $\begin{array}{c}\text { TEMP max } \\
\text { annual mean }\end{array}$ & $\begin{array}{c}\text { NDVI } \\
\text { Built-up areas }\end{array}$ & $\begin{array}{c}\text { NDVI Forest } \\
\text { cover }\end{array}$ & $\begin{array}{c}\text { NDVI } \\
\text { Crop }\end{array}$ & $\begin{array}{c}\text { NDVI } \\
\text { Bare }\end{array}$ & $\begin{array}{c}\text { NDVI } \\
\text { Grass }\end{array}$ & $\begin{array}{c}\text { NDVI } \\
\text { Shrub }\end{array}$ \\
\hline 1987 & 1171 & 22.54 & 0.4 & 0.6 & 0.46 & 0.39 & 0.56 & 0.48 \\
1997 & 1038.6 & 23.48 & 0.34 & 0.53 & 0.35 & 0.33 & 0.42 & 0.41 \\
2007 & 1139.6 & 22.03 & 0.44 & 0.75 & 0.6 & 0.4 & 0.59 & 0.57 \\
2017 & 692.4 & 24.77 & 0.25 & 0.42 & 0.39 & 0.19 & 0.4 & 0.39 \\
Temp Pearson Correlation (R) & & & -0.9996 & -0.9561 & -0.767 & -0.9768 & -0.9311 & -0.9044 \\
PPR Pearson Correlation (R) & & & 0.94116 & 0.82062 & 0.51515 & 0.99258 & 0.80082 & 0.71443 \\
\hline
\end{tabular}

$=0.51$ ). Pearson correlation coefficient was very high between annual mean precipitation and forest, shrub, built-up, crop, bare land and grass land NDVI at $0.81 ; 0.97,0.70,0.68,0.813$, and 0.90 , respectively. Shrub lands had the highest NDVI followed by grassland NDVI, while crop lands had the least. Precipitation thus affected NDVI positively in Amala sub-catchment. Regarding the Pearson correlation coefficient between maximum mean temperature and land cover, forest land, shrub land, built-up areas, cropland, bare land and grassland NDVI were $-0.97,-0.93,-0.96,-0.94,-0.99$, and -0.99 , respectively, implying that temperature affected NDVI negatively in Amala sub-catchment. Temperature showed less variation $\left(\mathrm{R}^{2}=0.32\right)$ than precipitation $\left(\mathrm{R}^{2}=0.51\right)$. Generally, the findings point to a strong impact of climate on land cover categories in Amala sub-catchment.

The mean total annual precipitation was high, while maximum mean annual temperature was low with respect to the NDVI of all land cover categories (see year 2007) (Table 5, Figure 4). On the contrary, when maximum mean annual temperature was high and mean total annual precipitation was low, NDVI of all land cover categories was lowest (see year 2017). Generally, from 1987 to 2017 mean total annual precipitation decreased from 1033.95 to $660.01 \mathrm{~mm}$ - a decrease of $-373.94 \mathrm{~mm}$ which is by $-36.17 \%$. Over the same period, maximum mean annual temperature increased from $24.13^{\circ} \mathrm{C}$ to $26.96^{\circ} \mathrm{C}$; an increase of $2.83^{\circ} \mathrm{C}$ which translates to $11.73 \%$ increase. Changes in maximum mean annual temperature $\left(\mathrm{R}^{2}=0.32\right)$ and mean total annual precipitation $\left(\mathrm{R}^{2}=0.51\right)$ have thus led to changes in land cover categories differently (Tables 5-7).

\subsubsection{Sand River Sub-Catchment}

Sand river sub-catchment showed varying trends in NDVI of different land cover 


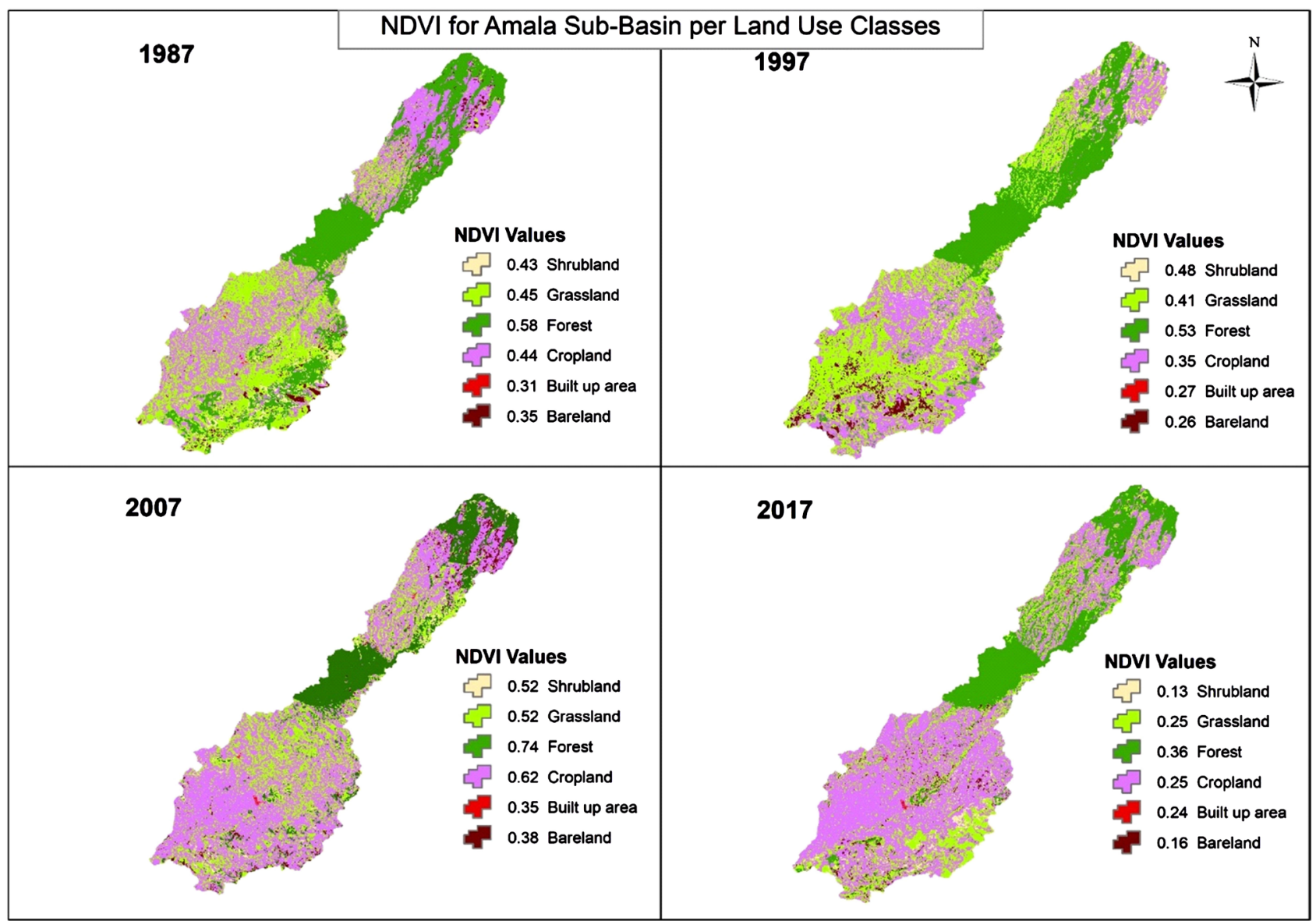

Figure 4. October land cover categories NDVI from 1987 to 2017 in Amala sub-catchment.

Table 5. NDVI per land cover category in Amala sub-catchment 1987-2017.

\begin{tabular}{|c|c|c|c|c|c|c|c|c|c|c|c|c|}
\hline YEAR & $\begin{array}{c}\text { NDVI } \\
1987\end{array}$ & $\begin{array}{c}\text { NDVI } \\
1997\end{array}$ & $\begin{array}{c}\text { NDVI } \\
\text { change } \\
1987 \text { to } \\
1997\end{array}$ & $\begin{array}{c}\text { NDVI \% } \\
\text { change } \\
1987 \text { to } \\
1997\end{array}$ & $\begin{array}{c}\text { NDVI } \\
2007\end{array}$ & $\begin{array}{c}\text { NDVI } \\
\text { change } \\
1997 \text { to } \\
2007\end{array}$ & $\begin{array}{c}\text { NDVI \% } \\
\text { change } \\
1997 \text { to } \\
2007\end{array}$ & $\begin{array}{c}\text { NDVI } \\
2017\end{array}$ & $\begin{array}{l}\text { NDVI } \\
\text { Change } \\
2007 \text { to } \\
2017\end{array}$ & $\begin{array}{c}\text { NDVI \% } \\
\text { change } \\
2007 \text { to } \\
2017\end{array}$ & $\begin{array}{c}\text { NDVI } \\
\text { Change } \\
1987 \text { to } \\
2017\end{array}$ & $\begin{array}{c}\text { NDVI \% } \\
\text { change } \\
1987-2017\end{array}$ \\
\hline FOREST & 0.58 & 0.53 & -0.05 & -8.6 & 0.74 & 0.21 & 39.62 & 0.36 & -0.38 & -51.35 & -0.22 & -37.9 \\
\hline SHRUB & 0.43 & 0.48 & 0.05 & 11.6 & 0.57 & 0.09 & 18.75 & 0.13 & -0.44 & -77.19 & -0.3 & -69.8 \\
\hline BUILT & 0.31 & 0.27 & -0.04 & -12.9 & 0.35 & 0.08 & 29.63 & 0.24 & -0.11 & -31.43 & -0.07 & -22.6 \\
\hline CROP & 0.44 & 0.35 & -0.09 & -20.5 & 0.62 & 0.27 & 77.14 & 0.25 & -0.37 & -59.68 & -0.19 & -43.2 \\
\hline BARE & 0.35 & 0.26 & -0.09 & -25.7 & 0.38 & 0.12 & 46.15 & 0.16 & -0.22 & -57.89 & -0.19 & -54.3 \\
\hline GRASS & 0.45 & 0.41 & -0.04 & -8.9 & 0.52 & 0.11 & 26.83 & 0.25 & -0.27 & -51.92 & -0.2 & -44.4 \\
\hline
\end{tabular}

Table 6. Trend of rainfall and temperature.

\begin{tabular}{|c|c|c|c|c|c|c|c|c|c|c|c|c|}
\hline YEAR & 1987 & 1997 & $\begin{array}{c}\text { Change } \\
\text { btw } 1987 \\
\& 1997\end{array}$ & $\begin{array}{c}\text { \% change } \\
\text { btw } 1987 \\
\text { \& } 1997\end{array}$ & 2007 & $\begin{array}{c}\text { Change } \\
\text { btw } 1997 \\
\& 2007\end{array}$ & $\begin{array}{c}\text { \% change } \\
\text { btw } 1997 \\
\& 2007\end{array}$ & 2017 & $\begin{array}{l}\text { Change } \\
\text { btw } 2007 \\
\& 2017\end{array}$ & $\begin{array}{c}\text { \% change } \\
\text { btw } 2007 \\
\text { to } 2017\end{array}$ & $\begin{array}{l}\text { Change } \\
\text { btw } 1987 \\
\text { \& } 2017\end{array}$ & $\begin{array}{c}\text { \% change } \\
\text { btw } 1987 \\
\text { to } 2017\end{array}$ \\
\hline PPT annual mean & 1033.9 & 1098.9 & 64.9 & 6.28 & 1079.6 & -19.24 & -1.75 & 660.0 & -419.6 & -38.87 & -373.94 & -36.17 \\
\hline $\begin{array}{l}\text { TEMP max } \\
\text { annual mean }\end{array}$ & 24.13 & 25.05 & 0.92 & 3.81 & 23.35 & -1.7 & -6.79 & 26.96 & 3.61 & 15.46 & 2.83 & 11.728 \\
\hline
\end{tabular}


categories in the dry season (October) from week $\left(\mathrm{R}^{2}=0.19\right)$ to moderate $(0.33$ to 0.5$)$. Changes in mean bare land NDVI of $R^{2}=0.5$; shrub land NDVI of $R^{2}=$ 0.36 ; forest land cover NDVI of $R^{2}=0.0 .33$; and grassland NDVI of $R^{2}=0.19$ were observed. Greater NDVI changes of $\mathrm{R}^{2}=0.5$ were observed in bare land within Sand river sub-catchment. Temperature also showed relative low change in mean $R^{2}=0.22$ while rainfall showed an even lower change in mean $R^{2}=0.06$ (Tables 8-10, Figure 5). Nevertheless, Pearson's correlation coefficient was very high between annual mean precipitation and different land cover categories i.e. forest cover, bare land, shrub land and grassland whose NDVIs were 0.75, 0.79, 0.76 and 0.78 respectively. The highest being observed in bare land was followed by grassland NDVI, while the least was observed in forest land. Regarding Pearson's correlation coefficient between maximum mean temperature and different land cover categories, i.e. forest cover, bare land, shrub land and grassland, NDVIs were $-0.96,-0.87,-0.95$, and -0.971 , respectively. Generally, NDVI was affected

Table 7. Person correlation between temperature, precipitation and NDVI land cover categories between 1987-2017 in Amala sub-catchment.

\begin{tabular}{cccccccccc}
\hline \multirow{2}{*}{ YEAR } & \multicolumn{2}{c}{ Total Annual Mean annual } & Forest & Shrub & Built & Crop & Bare land & Grassland \\
& Rainfall & max Temp & & & & & & & \\
1987 & 1033.95 & 24.13 & 0.58 & 0.43 & 0.31 & 0.44 & 0.35 & 0.45 \\
1997 & 1098.85 & 25.05 & 0.53 & 0.48 & 0.27 & 0.35 & 0.26 & 0.41 \\
2007 & 1079.61 & 23.35 & 0.74 & 0.57 & 0.35 & 0.62 & 0.38 & 0.52 \\
2017 & 660.01 & 26.96 & 0.36 & 0.13 & 0.24 & 0.25 & 0.16 & 0.25 \\
Temp Pearson Correlation (R) & & & -0.9746 & -0.9334 & -0.95854 & -0.93628 & -0.99078 & -0.99485 \\
Ppt Pearson Correlation (R) & & & 0.81299 & 0.966716 & 0.698927 & 0.684236 & 0.813424 & 0.902623 \\
\hline
\end{tabular}

Table 8. NDVI per land cover category within Sand River sub-catchment 1987-2017.

\begin{tabular}{|c|c|c|c|c|c|c|c|c|c|c|c|c|}
\hline YEAR & $\begin{array}{c}\text { NDVI } \\
1987\end{array}$ & $\begin{array}{c}\text { NDVI } \\
1997\end{array}$ & $\begin{array}{c}\text { NDVI } \\
\text { change btw } \\
1987 \& \\
1997\end{array}$ & $\begin{array}{c}\text { NDVI \% } \\
\text { change btw } \\
1987 \& \\
1997\end{array}$ & $\begin{array}{c}\text { NDVI } \\
2007\end{array}$ & $\begin{array}{c}\text { NDVI } \\
\text { change } \\
1997 \text { to } \\
2007\end{array}$ & $\begin{array}{c}\text { NDVI \% } \\
\text { change btw } \\
1997 \& \\
2007\end{array}$ & $\begin{array}{c}\text { NDVI } \\
2017\end{array}$ & $\begin{array}{c}\text { NDVI } \\
\text { change btw } \\
2007 \& \\
2017\end{array}$ & $\begin{array}{l}\text { NDVI \% } \\
\text { change btw } \\
2007 \& \\
2017\end{array}$ & $\begin{array}{c}\text { NDVI } \\
\text { change } \\
1987 \text { to } \\
2017\end{array}$ & $\begin{array}{c}\text { NDVI \% } \\
\text { change btw } \\
1987 \& \\
2017\end{array}$ \\
\hline Forest & 0.37 & 0.39 & 0.02 & 5.4 & 0.42 & 0.03 & 7.69 & 0.25 & -0.17 & -40.48 & -0.12 & -32.4 \\
\hline Bare & 0.23 & 0.25 & 0.02 & 8.7 & 0.24 & -0.01 & -4.00 & 0.15 & -0.09 & -37.50 & -0.08 & -34.8 \\
\hline Shrub & 0.29 & 0.31 & 0.02 & 6.9 & 0.33 & 0.02 & 6.45 & 0.18 & -0.15 & -45.45 & -0.11 & -37.9 \\
\hline Grass & 0.28 & 0.31 & 0.03 & 10.7 & 0.35 & 0.04 & 12.90 & 0.19 & -0.16 & -45.71 & -0.09 & -32.1 \\
\hline
\end{tabular}

Table 9. Trend of rainfall and temperature 1987-2017.

\begin{tabular}{|c|c|c|c|c|c|c|c|c|c|c|c|c|}
\hline YEAR & 1987 & 1997 & $\begin{array}{c}\text { change } \\
\text { btw } 1987 \\
\& 1997\end{array}$ & $\begin{array}{c}\text { \% change } \\
\text { btw } 1987 \\
\& 1997\end{array}$ & 2007 & $\begin{array}{l}\text { Change } \\
\text { btw } 1997 \\
\text { to } 2007\end{array}$ & $\begin{array}{c}\text { \% change } \\
\text { btw } 1997 \\
\text { to } 2007\end{array}$ & 2017 & $\begin{array}{c}\text { Change } \\
\text { btw } 2007 \\
\text { to } 2017\end{array}$ & $\begin{array}{c}\text { \% change } \\
\text { btw } 2007 \\
\text { to } 2017\end{array}$ & $\begin{array}{c}\text { Change } \\
\text { btw } 1987 \\
\text { to } 2017\end{array}$ & $\begin{array}{c}\text { \% change } \\
\text { btw } 1987 \\
\text { to } 2017\end{array}$ \\
\hline $\begin{array}{l}\text { PPT annual } \\
\text { mean }\end{array}$ & 430.1 & 904.3 & 474.25 & 110.27 & 718.4 & -185.9 & -20.6 & 327.6 & -390.8 & -54.4 & -102.5 & -23.83 \\
\hline $\begin{array}{l}\text { TEMP max } \\
\text { annual mean }\end{array}$ & 24.3 & 24.4 & 0.17 & 0.70 & 23.3 & -1.08 & -4.4 & 25.9 & 2.6 & 11.0 & 1.66 & 6.85 \\
\hline
\end{tabular}


Table 10. Person correlation between temp, ppt and NDVI land cover categories 1987-2017.

\begin{tabular}{cccccccc}
\hline Year & PPT annual mean & TEMP max annual mean & Forest & Bare land & Shrub & Grass \\
\hline 1987 & 430.09 & 24.25 & 0.37 & 0.23 & 0.29 & 0.28 \\
1997 & 904.34 & 24.42 & 0.39 & 0.25 & 0.31 & 0.31 \\
2007 & 718.43 & 23.34 & 0.42 & 0.24 & 0.33 & 0.35 \\
2017 & 327.59 & 25.91 & 0.25 & 0.15 & 0.18 & 0.19 \\
Temp Pearson Correlation (R) & & & -0.96497 & -0.86935 & -0.9534 & -0.9681 \\
PPT Pearson Correlation (R) & & & 0.751871 & 0.794609 & 0.762858 & 0.778507 \\
\hline
\end{tabular}

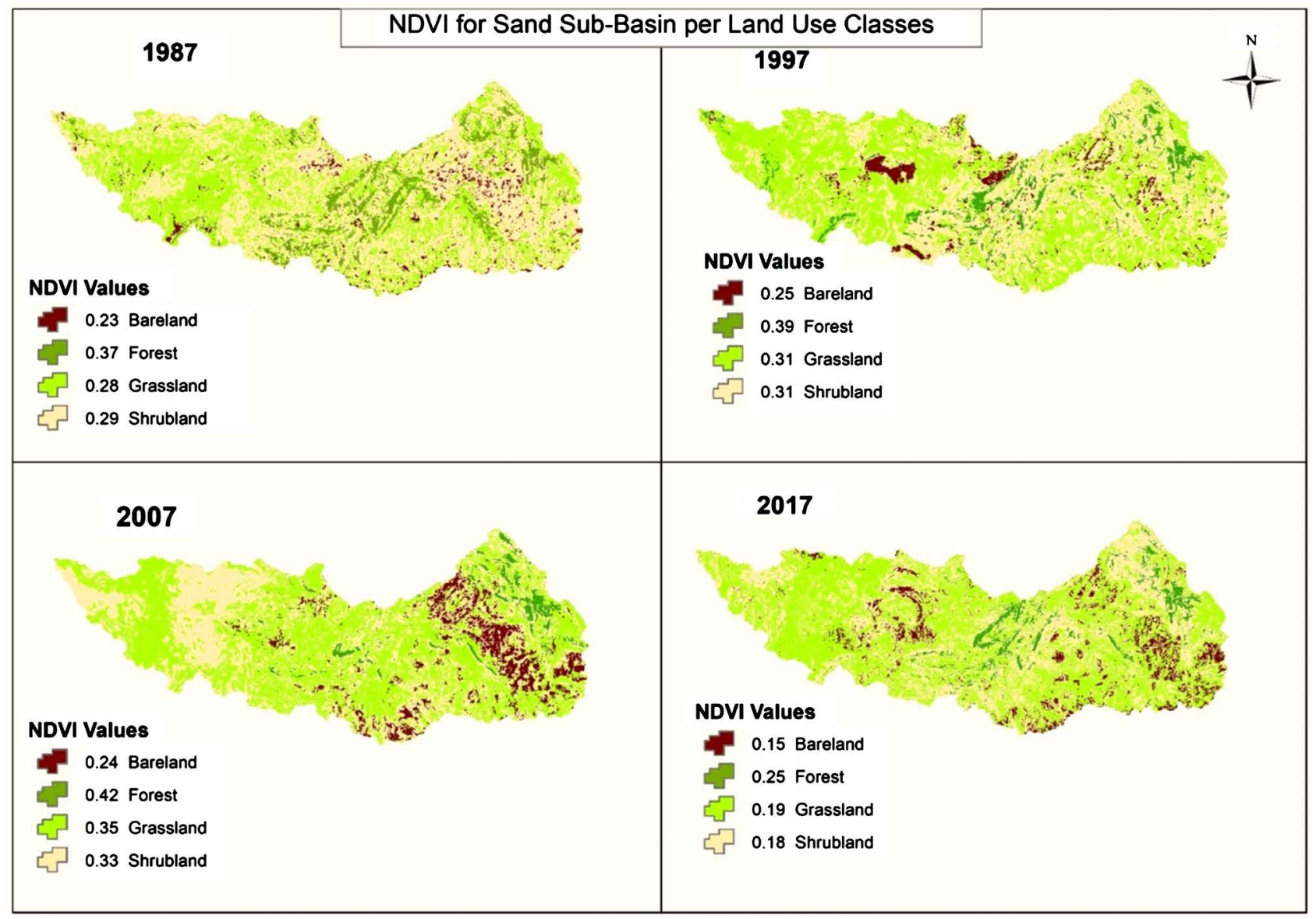

Figure 5. October land cover categories NDVI trend from 1987 to 2017 in Sand sub-catchment.

positively by precipitation and negatively by maximum temperature, though both temperature $\left(\mathrm{R}^{2}=0.22\right)$ and precipitation $\left(\mathrm{R}^{2}=0.06\right)$ showed low variation.

Findings showed that when mean total annual precipitation was high and maximum mean annual temperature low, NDVI of all land cover categories was high (see year 2007); and when maximum mean annual temperature was high and mean total annual precipitation was low, NDVI of all land cover categories was low (see year 2017). Mean total annual precipitation decreased from 430.09 $\mathrm{mm}$ to $327.59 \mathrm{~mm}$ - a decrease of $-102.5 \mathrm{~mm}$ translating to $-23.83 \%$ between 1987 and 2017, while maximum mean annual temperature increased from 
$24.25^{\circ} \mathrm{C}$ to $25.91^{\circ} \mathrm{C}$-translating to a $6.85 \%$ increase over the same period. Changes in maximum mean annual temperature $\left(\mathrm{R}^{2}=0.22\right)$ and mean total annual precipitation $\left(\mathrm{R}^{2}=0.06\right)$ have affected land cover categories differently in Sand River sub-catchment.

\subsubsection{Talek Sub-Catchment}

Varying trends in NDVI of land cover categories from weak $\left(\mathrm{R}^{2}=0.006\right.$ to 0.04$)$ to moderate ( 0.33 to 0.5$)$ levels were also observed in Talek sub-catchment over time during the dry season (October). Change in mean grassland cover category NDVI of $\mathrm{R}^{2}=0.006$; shrub land NDVI of $\mathrm{R}^{2}=0.0 .03$; forest cover NDVI of $\mathrm{R}^{2}=$ 0.04 ; bare land NDVI of $\mathrm{R}^{2}=0.45$ and crop land NDVI of $\mathrm{R}^{2}=0.6$, were observed. Greatest changes $\left(\mathrm{R}^{2}=0.6\right)$ were observed in cop lands. Temperature showed relatively low change in mean $\left(\mathrm{R}^{2}=0.24\right)$ while rainfall showed even lower change in mean $\left(\mathrm{R}^{2}=0.05\right)$. Pearson correlation coefficient between annual mean precipitation and land cover categories was moderate i.e. forest, crop, bare, grass and shrub, whose NDVIs were 0.67, 0.8, 0.42, 0.49 and 0.49 , respectively. The highest change was observed in crop land NDVI followed by forest land NDVI, while the least was observed in bare land NDVI. Pearson correlation coefficient between maximum mean temperature and land cover categories i.e. forest cover, crop land, bare land, grass land and shrub land NDVI were -0.80 , $0.90,-0.92,-0.79,-0.48$ respectively. Precipitation affected NDVI of different land cover categories positively while maximum temperature affected NDVI of different land cover categories negatively except crop lands in Talek sub-catchment. While temperature showed less variation $\left(\mathrm{R}^{2}=0.24\right)$ compared to precipitation $\left(\mathrm{R}^{2}=0.5\right)$ in Talek sub-catchment, correlation analysis between land cover categories NDVI and climate variables (temperature and precipitation) revealed a strong relationship between climatic factors and different land cover categories.

Generally, when mean total annual precipitation was high and maximum mean annual temperature was low, the NDVI of all land cover categories was high (see year 2007); and when maximum mean annual temperature was high and mean total annual precipitation was low, NDVI of all land cover categories was low (see year 2017). Mean total annual precipitation decreased from 773.24 $\mathrm{mm}$ to $429.28 \mathrm{~mm}$ - a decrease of $-343.96 \mathrm{~mm}$ which translates to $-44.48 \%$ between 1987 and 2017, while maximum annual mean temperature increased from $24.25^{\circ} \mathrm{C}$ to $25.91^{\circ} \mathrm{C}$; an increase of $1.66^{\circ} \mathrm{C}$ which translates to $6.85 \%$ over the same period. As a result, change in maximum mean annual temperature $\left(\mathrm{R}^{2}=\right.$ $0.24)$ and mean total annual precipitation $\left(\mathrm{R}^{2}=0.05\right)$ has affected land cover categories differently within Talek sub-catchment (Tables 11-13; and Figure 6).

\subsection{Transition Probability Matrix for Nyangores, Amala, Sand and Talek Sub-Catchments}

On the overall, the transitional probability matrix of the four sub-basins showed an interesting pattern (Table 14). Row categories represent land cover classes in 


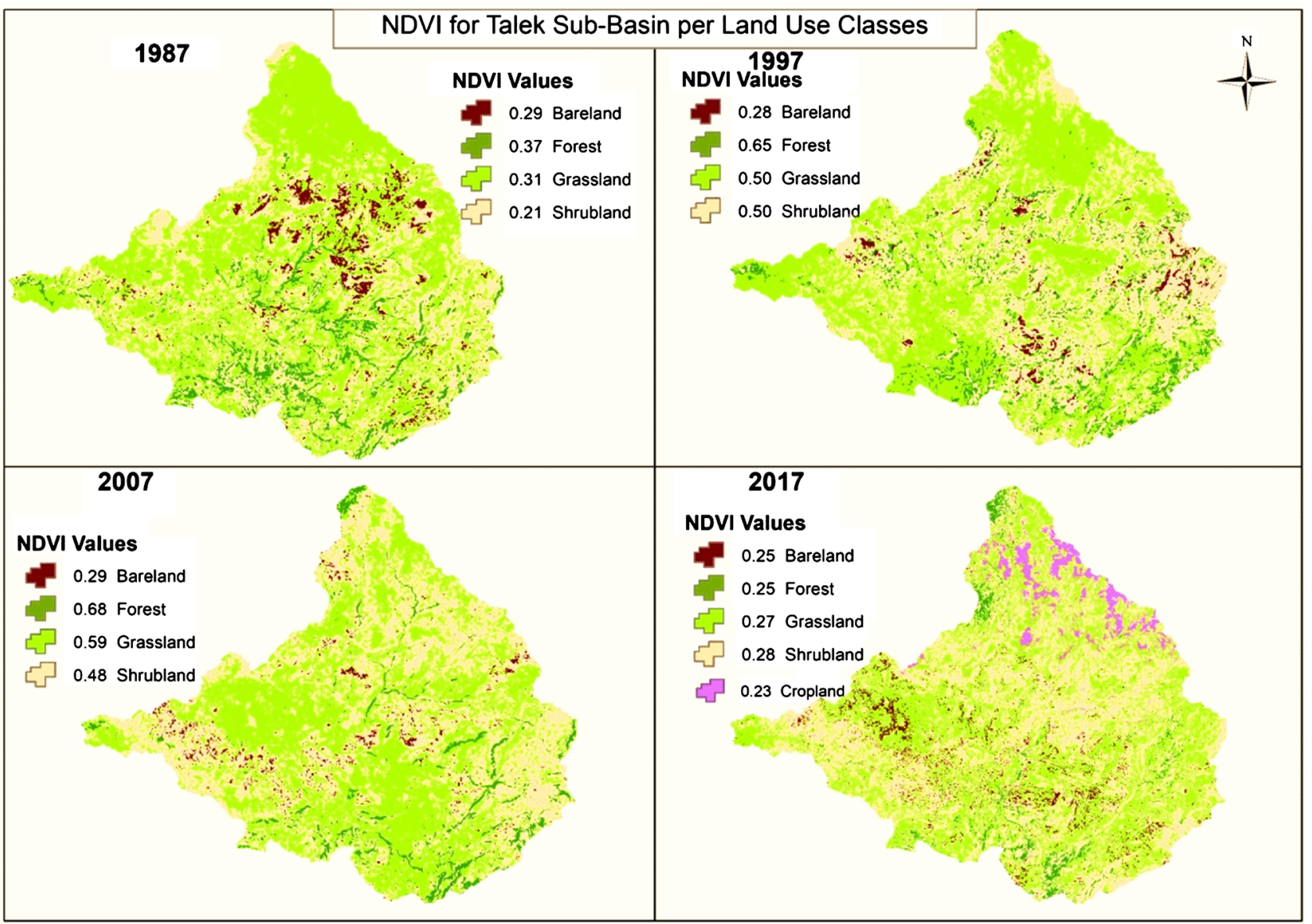

Figure 6. Land cover categories NDVI trend from 1987 to 2017 (Oct) in Talek sub-catchment.

Table 11. NDVI per land cover category within Talek sub-catchment 1987-2017.

\begin{tabular}{|c|c|c|c|c|c|c|c|c|c|c|c|c|}
\hline Year & $\begin{array}{c}\text { NDVI } \\
1987\end{array}$ & $\begin{array}{c}\text { NDVI } \\
1997\end{array}$ & $\begin{array}{c}\text { NDVI } \\
\text { change } \\
1987 \text { to } \\
1997\end{array}$ & $\begin{array}{c}\text { NDVI \% } \\
\text { change } \\
1987 \text { to } \\
1997\end{array}$ & $\begin{array}{c}\text { NDVI } \\
2007\end{array}$ & $\begin{array}{c}\text { NDVI } \\
\text { change } \\
1997 \text { to } \\
2007\end{array}$ & $\begin{array}{c}\text { NDVI \% } \\
\text { change } \\
1997 \text { to } \\
2007\end{array}$ & $\begin{array}{c}\text { NDVI } \\
2017\end{array}$ & $\begin{array}{c}\text { NDVI } \\
\text { change } \\
2007 \text { to } \\
2017\end{array}$ & $\begin{array}{c}\text { NDVI \% } \\
\text { change } \\
2007 \text { to } \\
2017\end{array}$ & $\begin{array}{c}\text { NDVI } \\
\text { change } \\
1987 \text { to } \\
2017\end{array}$ & $\begin{array}{c}\text { NDVI \% } \\
\text { change } \\
1987 \text { to } \\
2017\end{array}$ \\
\hline Forest & 0.37 & 0.65 & 0.28 & 75.7 & 0.68 & 0.03 & 4.62 & 0.25 & -0.43 & -63.24 & -0.12 & -32.4 \\
\hline Crop & 0 & 0 & 0 & 0.0 & 0 & 0 & 0.00 & 0.23 & 0.23 & 0.00 & 0.23 & 0.0 \\
\hline Bare & 0.29 & 0.28 & -0.01 & -3.4 & 0.29 & 0.01 & 3.57 & 0.27 & -0.02 & -6.90 & -0.02 & -6.9 \\
\hline Grass & 0.31 & 0.5 & 0.19 & 61.3 & 0.59 & 0.09 & 18.00 & 0.27 & -0.32 & -54.24 & -0.04 & -12.9 \\
\hline Shrub & 0.21 & 0.5 & 0.29 & 138.1 & 0.48 & -0.02 & -4.00 & 0.28 & -0.2 & -41.67 & 0.07 & 33.3 \\
\hline
\end{tabular}

Table 12. Trend of rainfall and temperature within Talek Sub-catchment 1987-2017.

\begin{tabular}{|c|c|c|c|c|c|c|c|c|c|c|c|c|}
\hline Year & 1987 & 1997 & $\begin{array}{l}\text { Change } \\
\text { btw } 1987 \\
\text { \& } 1997\end{array}$ & $\begin{array}{c}\text { \% change } \\
\text { btw } 1987 \\
\text { \& } 1997\end{array}$ & 2007 & $\begin{array}{l}\text { Change } \\
\text { btw } 1997 \\
\& 2007\end{array}$ & $\begin{array}{c}\text { \% change } \\
\text { btw } 1997 \\
\& 2007\end{array}$ & 2017 & $\begin{array}{c}\text { Change } \\
\text { btw } 2007 \\
\& 2017\end{array}$ & $\begin{array}{c}\text { \% Change } \\
\text { btw } 2007 \text { \& } \\
2017\end{array}$ & $\begin{array}{l}\text { Change } \\
\text { btw } 1987 \\
\& 2017\end{array}$ & $\begin{array}{c}\text { \% change } \\
\text { btw } 1987 \\
\& 2017\end{array}$ \\
\hline $\begin{array}{l}\text { PPT annual } \\
\text { mean }\end{array}$ & 773.24 & 1027.1 & 253.88 & 32.83 & 678.1 & -349.07 & -33.99 & 429.28 & -248.77 & -36.69 & -343.96 & -44.48 \\
\hline $\begin{array}{l}\text { TEMP max } \\
\text { annual mean }\end{array}$ & 24.25 & 24.42 & 0.17 & 0.70 & 23.4 & -1.02 & -4.18 & 25.91 & 2.51 & 10.73 & 1.66 & 6.85 \\
\hline
\end{tabular}


Table 13. Person correlation between temp, ppt and NDVI of land cover categories between 1987 \& 2017 in Talek sub-catchment.

\begin{tabular}{ccccccccc}
\hline Year & PPT annual mean & TEMP max annual mean & Forest & Crop & Bare & Grass & Shrub \\
\hline 1987 & 773.24 & 24.25 & 0.37 & 0 & 0.29 & 0.31 & 0.21 \\
1997 & 1027.12 & 24.42 & 0.65 & 0 & 0.28 & 0.5 & 0.5 \\
2007 & 678.05 & 23.4 & 0.68 & 0 & 0.29 & 0.59 & 0.48 \\
2017 & 429.28 & 25.91 & 0.25 & 0.23 & 0.27 & 0.27 & 0.28 \\
Temp Pearson Correlation (R) & & & -0.80239 & 0.903978 & -0.91915 & -0.79001 & -0.48255 \\
PPR Pearson Correlation (R) & & & 0.668472 & -0.80288 & 0.415691 & 0.488346 & 0.494804 \\
\hline
\end{tabular}

Table 14. Transitional probability table derived from the land use land cover map of 2007.

\begin{tabular}{lcccccc}
\hline \multicolumn{1}{l}{ Amala 2007-2017 Transition Potential } & \multicolumn{1}{c}{} \\
\hline \multicolumn{1}{c}{ FROM/TO } & 1 Forest & 2 Grassland & 3 Shrub Land & 4 Cropland & 5 Bare Land & 6 Built up areas \\
\hline 1. Forest & 0.808587 & 0.089546 & 0.012824 & 0.080526 & 0.009 & 0.00 \\
2. Grassland & 0.453074 & 0.260371 & 0.072374 & 0.197999 & 0.016 & 0.00 \\
3. Shrub Land & 0.125763 & 0.288336 & 0.029044 & 0.532872 & 0.024 & 0.00 \\
4. Cropland & 0.038497 & 0.233278 & 0.013664 & 0.69706 & 0.018 & 0.00 \\
5. Bare Land & 0.054522 & 0.236698 & 0.008758 & 0.680972 & 0.019 & 0.00 \\
6. Built up areas & 0.00 & 0.00 & 0.00 & 0.00 & 0.00 & 1.00 \\
\hline
\end{tabular}

Nyangores sub-catchment 2007-2017 Transition Potential

\begin{tabular}{lcccccc}
\hline \multicolumn{1}{c}{ FROM/TO } & 1 Forest & 2 Grassland & 3 Shrub Land & 4 Cropland & 5 Bare Land & 6 Built up areas \\
\hline 1. Forest & 0.656541 & 0.143995 & 0.019081 & 0.170156 & 0.010065 & 0.000161 \\
2. Grassland & 0.044171 & 0.118876 & 0.004942 & 0.72793 & 0.102007 & 0.002074 \\
3. Shrub Land & 0.030252 & 0.142379 & 0.0005945 & 0.730669 & 0.0868804 & 0.003951 \\
4. Cropland & 0.041294 & 0.08228 & 0.002465 & 0.822188 & 0.051772 & 0.000000 \\
5. Bare Land & 0.002294 & 0.097095 & 0.000000 & 0.761468 & 0.13685 & 0.0002294 \\
6. Built up areas & 0.008130 & 0.044715 & 0.000000 & 0.182927 & 0.01626 & 0.7479670 \\
\hline
\end{tabular}

Sand River sub-catchment 2007-2017 Transition Potential

\begin{tabular}{lcccc}
\hline \multicolumn{1}{c}{ FROM/TO } & 1. Forest & 2. Grassland & 3. Shrub Land & 4. Bare Land \\
\hline 1. Forest & 0.571987 & 0.376739 & 0.05018 & 0.001094 \\
2. Grassland & 0.043466 & 0.370093 & 0.529799 & 0.056641 \\
3. Shrub Land & 0.017485 & 0.40707 & 0.53481 & 0.040635 \\
4. Bare Land & 0.00 & 0.221829 & 0.478519 & 0.299652 \\
\hline
\end{tabular}

Talek sub-catchment 2007-2017 Transition Potential

\begin{tabular}{lcccc}
\hline \multicolumn{1}{c}{ FROM/TO } & 1. Forest & 2. Grassland & 3. Shrub Land & 4. Bare Land \\
\hline 1. Forest & 0.755453 & 0.09977 & 0.144031 & 0.000738 \\
2. Grassland & 0.546455 & 0.017685 & 0.371694 & 0.064166 \\
3. Shrub Land & 0.372432 & 0.037934 & 0.501656 & 0.087977 \\
4. Bare Land & 0.717363 & 0.00026 & 0.228913 & 0.053465
\end{tabular}

NB: Crop land and built up in Sand and Talek sub-catchments is very minimal to be captured by this resolution of $90 \mathrm{~m}$. 
2007 whilst column categories represent 2017 classes. These dynamics helps to understand what happened to different land cover categories (what gained and lost and from which land cover category to another) and what is likely to change in the future. The 2007 and 2017 land cover maps were then used to predict the land cover change by 2027 (Table 14).

\subsection{Projected Land Cover Change between 2017 and 2027 in the Four Sub-Catchment}

\subsubsection{Projected Land Cover Change between 2017 and 2027 in Nyangores Sub-Catchment}

Over the 30-year period (1987-2017), significant changes in land cover were observed in Nyangores sub-catchment (Table 15). Findings show that between 1987 and 1997 grasslands decreased by $-13.96 \%$ and forest land by -0.33 , while all other land cover categories increased, i.e. shrub lands by $57.60 \%$, built-up areas by $38.25 \%$, crop land by $12.53 \%$ and bare land by $7.73 \%$. A similar trend was evident in the following decade (1997-2007) whereby shrub land decreased by $-33.04 \%$, grassland by $-8.84 \%$, forest land by $-0.95 \%$, while build up areas increased further by $22.85 \%$, crop land by $9.81 \%$ and bare land by $8.47 \%$. However, between 2007 and 2017, grass land decreased by $-26.32 \%$, shrub land by $-26.26 \%$ and forest cover by $-25.54 \%$, while crop land, bare land and built up area increased by $58.21 \%, 39.75 \%$ and $31.55 \%$, respectively. Overall, land cover change between 1987 and 2017 showed a significant increase in crop land by $95.51 \%$, bare land by $63.31 \%$ and built-up areas by $123.42 \%$; at the expense of shrub land $(-22.19 \%)$, forest cover $(-26.48 \%)$ and grass land $(-42.21 \%)$ in Nyangores sub-catchment. The findings further showed that the greatest degradation of forests, shrubs and grasslands occurred between 1987 and 2007 (Tables 15-17 and Figure 7, Figure 8).

\subsubsection{Projected Land Cover Change between 2017 and 2027 in Amala Sub-Catchment}

In the Amala sub-catchment, significant changes were observed in land cover classes over the 30-year period (1987-2017) (Figures 9-11). Some land cover categories increased over time at the expense of others while some decreased (Table 18). For instance, bare land decreased by $-62.63 \%$, shrub lands by $-45.82 \%$

Table 15. Land cover change for 1987, 1997 and 2007 in Nyangores sub-catchment.

\begin{tabular}{cccccc}
\hline Sub-catchment & Category & $\begin{array}{c}1987 \text { Area } \\
(\mathrm{Ha})\end{array}$ & $\begin{array}{c}1997 \text { Area } \\
(\mathrm{Ha})\end{array}$ & $\begin{array}{c}2007 \text { Area } \\
(\mathrm{Ha})\end{array}$ & $\begin{array}{c}2017 \text { Area } \\
(\mathrm{Ha})\end{array}$ \\
\hline \multirow{5}{*}{ Nyangores } & Forest & $41,031(43.7 \%)$ & $40,897(43.8 \%)$ & $40,510(43.6 \%)$ & $30,164(32.3 \%)$ \\
& Grassland & $27,681(29.5 \%)$ & $23,817(25.5 \%)$ & $21,712(23.3 \%)$ & $15,997(17.1 \%)$ \\
& Shrub Land & $1014(1.1 \%)$ & $1598(1.7 \%)$ & $1070(1.2 \%)$ & $789(0.8 \%)$ \\
& Cropland & $21,835(23.3 \%)$ & $24,572(26.3 \%)$ & $26,983(29.0 \%)$ & $42,690(45.7 \%)$ \\
& Bare Land & $2279(2.4 \%)$ & $2455(2.6 \%)$ & $2663(2.9 \%)$ & $3721.6(4.0 \%)$ \\
& Built up areas & $47.4(0.01 \%)$ & $65.53(0.1 \%)$ & $80.5(0.1 \%)$ & $105.9(0.1 \%)$ \\
\hline
\end{tabular}


Table 16. Land cover percentage changes by category per decade in Nyangores sub-catchment.

\begin{tabular}{ccccc}
\hline Category & $\begin{array}{c}\text { 1987-1997 } \\
\text { \% Change }\end{array}$ & $\begin{array}{c}\text { 1997-2007 } \\
\text { \% Change }\end{array}$ & $\begin{array}{c}\text { 2007-2017 } \\
\text { \% Change }\end{array}$ & $\begin{array}{c}\text { 1987-2017 } \\
\text { Overall \% change }\end{array}$ \\
\hline Forest & -0.33 & -0.95 & -25.54 & -26.48 \\
Grassland & -13.96 & -8.84 & -26.32 & -42.21 \\
Shrub Land & 57.60 & -33.04 & -26.26 & -22.19 \\
Cropland & 12.53 & 9.81 & 58.21 & 95.51 \\
Bare Land & 7.73 & 8.47 & 39.75 & 63.31 \\
Built up areas & 38.25 & 22.84 & 31.55 & 123.42 \\
\hline
\end{tabular}

Table 17. Projected land cover by 2027 in Nyangores sub-catchment.

\begin{tabular}{ccccccc}
\hline $\begin{array}{c}\text { Land cover } \\
\text { category }\end{array}$ & $\begin{array}{c}1987 \text { Area (Ha) } \\
\text { and \% of total area }\end{array}$ & $\begin{array}{c}1997 \text { Area (Ha) } \\
\text { and of total area }\end{array}$ & $\begin{array}{c}2007 \text { Area (Ha) } \\
\text { and of total area }\end{array}$ & $\begin{array}{c}\text { 2017 Area (Ha) } \\
\text { and \% of total area }\end{array}$ & $\begin{array}{c}\text { Projected land cover } \begin{array}{c}\% \text { change btw } 2017 \\
\text { by } 2027 \text { area (Ha) }\end{array} \\
\text { to 2027 (10 yrs) }\end{array}$ \\
\hline Forest & $41,031(43.7 \%)$ & $40,897(43.8 \%)$ & $40,510(43.6 \%)$ & $30,164(32.3 \%)$ & $32,688.6$ & $8.37 \%$ \\
Grassland & $27,681(29.5 \%)$ & $23,817(25.5 \%)$ & $21,712(23.3 \%)$ & $15,997(17.1 \%)$ & $20,636.13$ & $29.00 \%$ \\
Shrub Land & $1014(1.1 \%)$ & $1598(1.7 \%)$ & $1070(1.2 \%)$ & $789(0.8 \%)$ & 1673.5 & $112.10 \%$ \\
Cropland & $21,835(23.3 \%)$ & $24,572(26.3 \%)$ & $26,983(29.0 \%)$ & $42,690(45.7 \%)$ & $34,761.97$ & $-18.57 \%$ \\
Bare Land & $2279(2.4 \%)$ & $2455(2.6 \%)$ & $2663(2.9 \%)$ & $3721.6(4.0 \%)$ & 3523.6 & $-5.32 \%$ \\
Built up areas & $47.4(0.01 \%)$ & $65.53(0.1 \%)$ & $80.5(0.1 \%)$ & $105.9(0.1 \%)$ & 183.6 & $73.37 \%$ \\
\hline
\end{tabular}

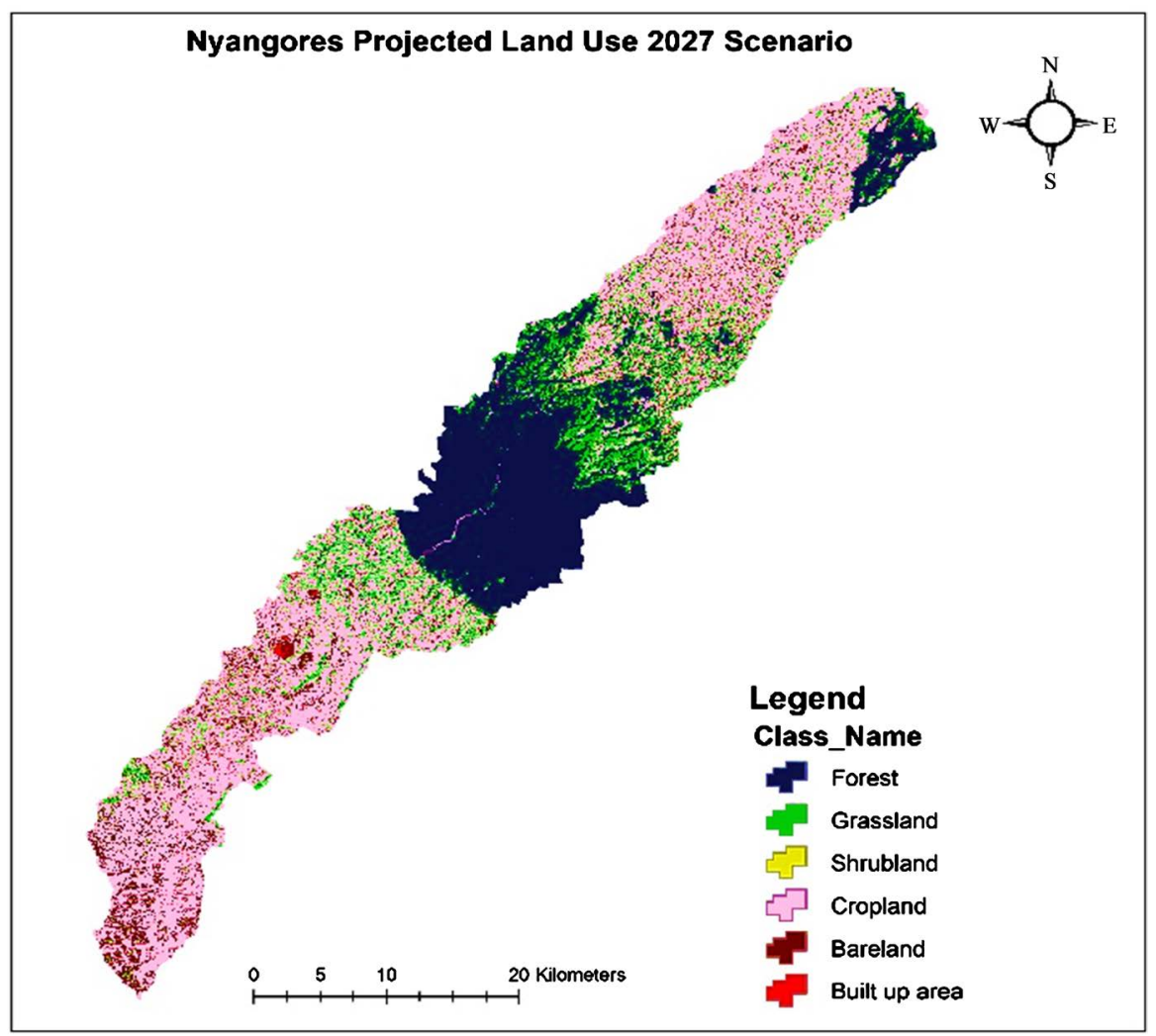

Figure 7. Map Predicted land cover change by 2027 within Nyangores sub-catchment. 


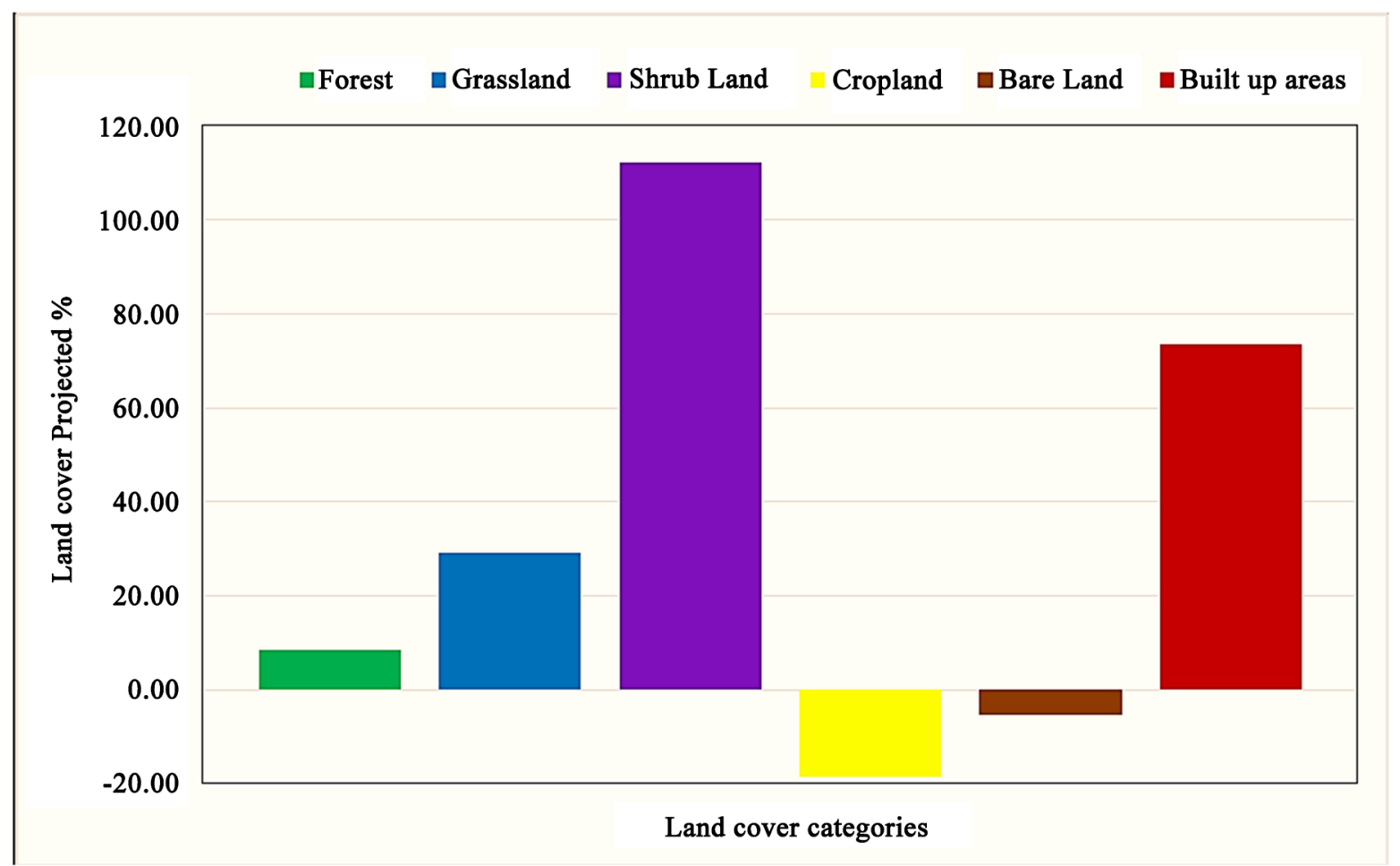

Figure 8. Projected land cover percentage change between 2017 and 2027 within Nyangores sub-catchment.

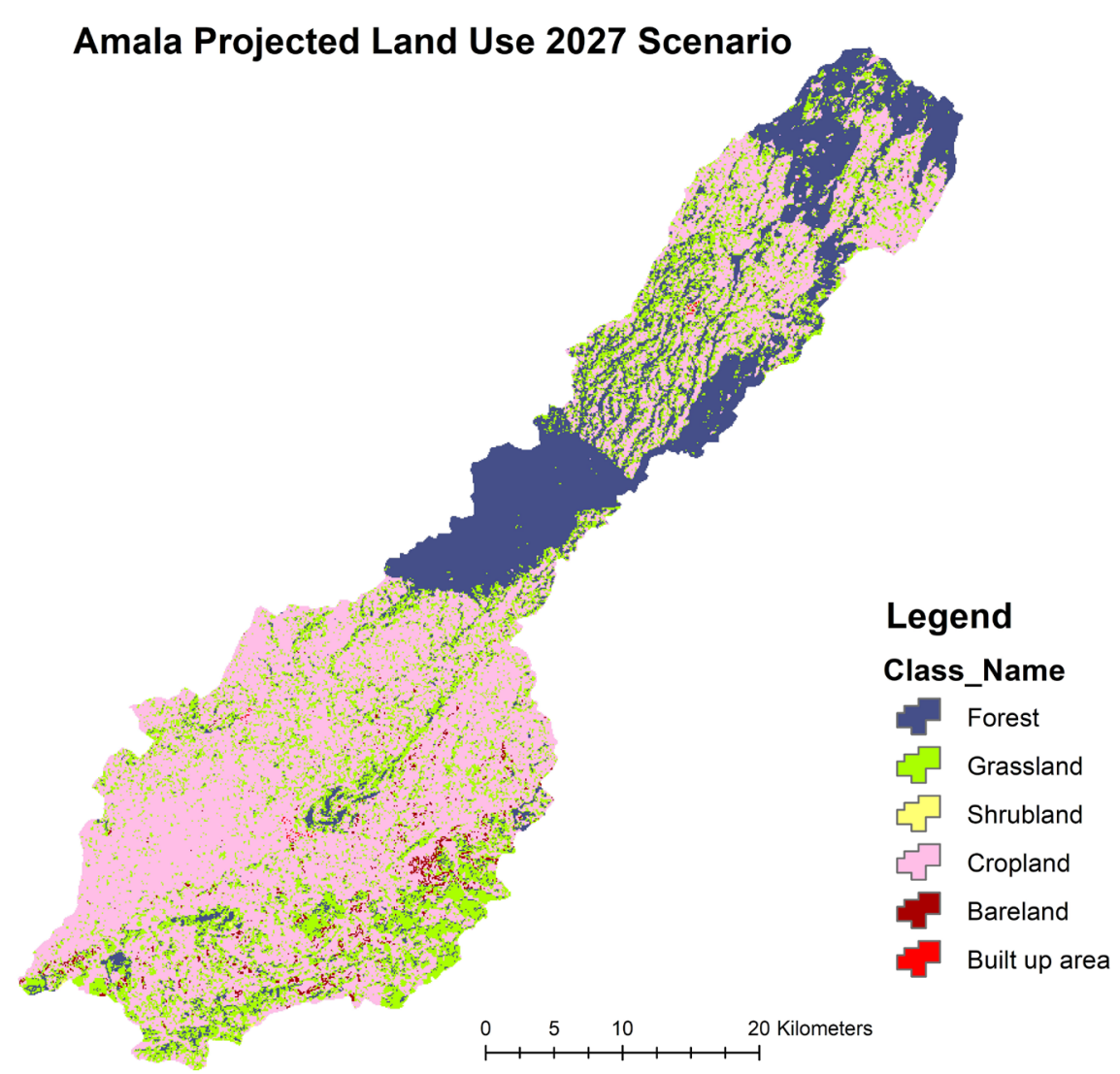

Figure 9. Predicted land cover change in Amala sub-catchment by 2027. 


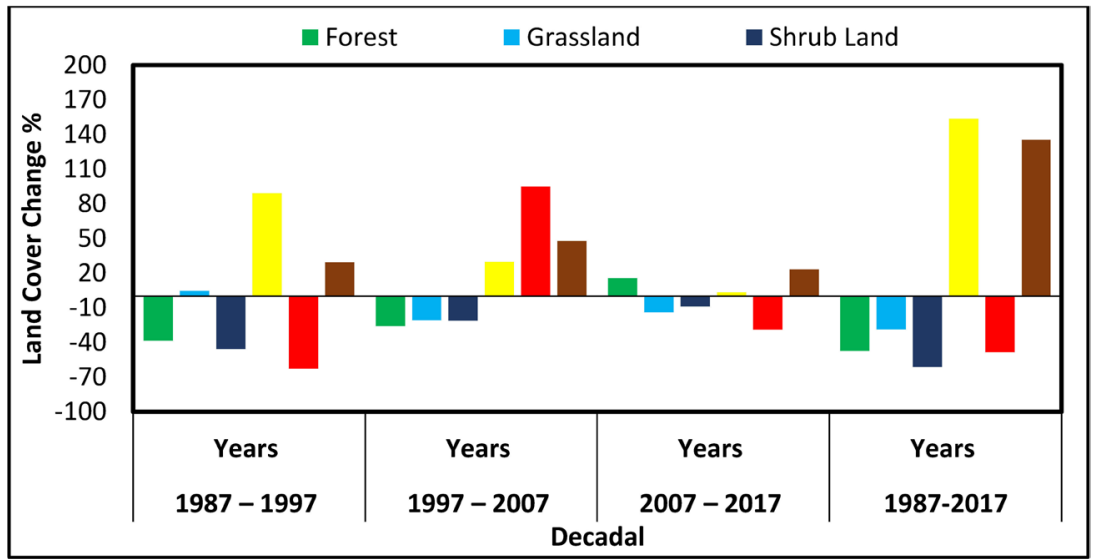

Figure 10. Summary decadal land cover change percentage for Amala sub-catchment.

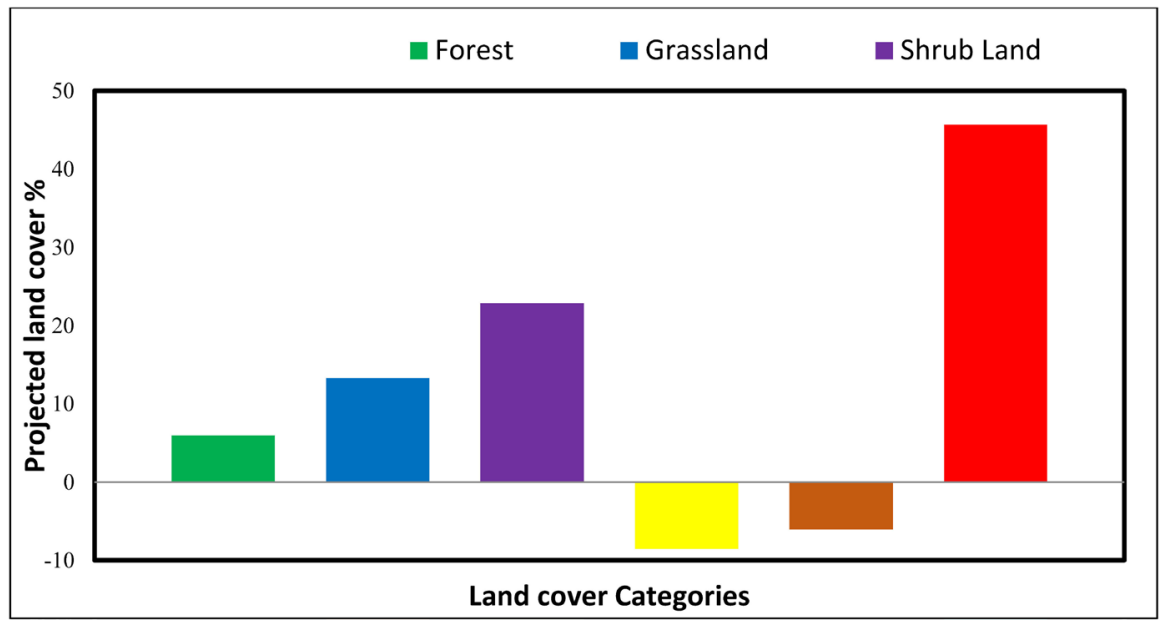

Figure 11. Projected land cover \% change between 2017 and 2027 in Amala sub-catchment.

Table 18. Land cover classes change for 1987, 1997, 2007 and 2017 in Amala sub-catchment.

\begin{tabular}{ccccc}
\hline Category & 1987 Area (Ha) & 1997 Area (Ha) & 2007 Area (Ha) & 2017 Area (Ha) \\
\hline Forest & $56,609(39.8 \%)$ & $34,830(24.4 \%)$ & $25,798(18.2 \%)$ & $29,784(21.0 \%)$ \\
Grassland & $44,269(31.1 \%)$ & $46,186(32.3 \%)$ & $36,625(25.8 \%)$ & $31,550(22.2 \%)$ \\
Shrub Land & $6418(4.5 \%)$ & $3477(2.4 \%)$ & $2738(1.9 \%)$ & $2495(1.8 \%)$ \\
Cropland & $29,828(21.0 \%)$ & $56,415(39.5 \%)$ & $73,168(51.5 \%)$ & $75,574(53.2 \%)$ \\
Bare Land & $5068(3.6 \%)$ & $1894(1.3 \%)$ & $3691(2.6 \%)$ & $2615(1.8 \%)$ \\
Built up areas & $34(0.02 \%)$ & $44(0.03 \%)$ & $65(0.05 \%)$ & $80(0.06 \%)$ \\
\hline
\end{tabular}

and forest land by $-38.47 \%$, between 1987 and 1997 . However, over the same period, crop lands increased by $89.13 \%$, built up areas by $29.42 \%$ and grassland by $4.33 \%$. Between 1997 and 2007, significant changes occurred as shown in Table 19. Over the 1997-2007 period, forest cover decreased by $-25.93 \%$, shrub lands by $-21.25 \%$ and grass land by $-20.70 \%$, while bare land increased by $94.88 \%$, build up areas by $47.73 \%$ and cropland by $29.70 \%$. Over the $2007-2017$ decade, bare land decreased by $-29.15 \%$, grass land by $-13.86 \%$ and shrub lands by $-8.88 \%$. On the 
contrary, built-up areas increased by $23.08 \%$, forest cover by $15.45 \%$; and crop land by $3.29 \%$. Over the 30 -year period, forest land decreased by $-47.39 \%$; grassland by -28.73 , shrub land by $-61.12 \%$ and bare land by $-48.40 \%$ while crop land increased by $135.29 \%$ within Amala sub-catchment (Table 20).

\subsubsection{Projected Land Cover Change between 2017 and 2027 in Sand River Sub-Catchment}

In the 30-year period (1987-2017) under study, significant land cover changes were observed within Sand River sub-catchment. Between 1987 and 1997, for instance, forest cover decreased by $-28.41 \%$, while shrub land and grasslands decreased slightly by $-1.71 \%$ and $-0.73 \%$, respectively. However, bare land increased by a massive 210.16\%. Likewise, between 1997 and 2007, forest land, grass land and shrub land decreased by $-18.09 \%,-5.39 \%$ and $-2.59 \%$, respectively, while bare land increased by $108.82 \%$. A similar trend was observed in the 2007-2017 period in which shrub-lands, forest land and grass land decreased by $-6.01 \%,-2.42 \%$ and $-2.35 \%$, respectively, while bare land increased by $41.34 \%$. Overall, bare land increased significantly by $815.42 \%$, from just 2510 ha in 1987 to 22,977 ha, in 2017 . The highest decline in forest cover was observed between 1987 and 1997, while the highest decline in grassland and shrub land was observed over the 1997-2017 period, while the projected land cover increase is highest in grassland and lowest in bare land (Tables 21-23) and (Figures 12-14). Since most of Sand River sub-catchment lies within Maasai Mara Game Reserve with scattered settlements (Maasai Bomas and hotels/tents), it was difficult for

Table 19. Land cover percentage changes by category per decade in Amala sub-catchment.

\begin{tabular}{ccccc}
\hline Category & $\begin{array}{c}1987-1997 \\
\text { \% Change }\end{array}$ & $\begin{array}{c}1997-2007 \\
\text { \% Change }\end{array}$ & $\begin{array}{c}\text { 2007-2017 } \\
\text { \% Change }\end{array}$ & $\begin{array}{c}\text { 1987-2017 } \\
\text { Overall \% change }\end{array}$ \\
\hline Forest & -38.47 & -25.93 & 15.45 & -47.39 \\
Grassland & 4.33 & -20.70 & -13.86 & -28.73 \\
Shrub Land & -45.82 & -21.25 & -8.88 & -61.12 \\
Cropland & 89.13 & 29.70 & 3.29 & 153.37 \\
Bare Land & -62.63 & 94.88 & -29.15 & -48.40 \\
Built up areas & 29.41 & 47.73 & 23.08 & 135.29 \\
\hline
\end{tabular}

Table 20. Projected land cover by 2027 within Amala sub-catchment.

\begin{tabular}{ccccccc}
\hline Category & 1987 Area (Ha) & 1997 Area (Ha) & 2007 Area (Ha) & 2017 Area (Ha) & $\begin{array}{c}\text { Projected land cover by } \\
\text { 2027 Area (Ha) }\end{array}$ & $\begin{array}{c}\text { \% change between } \\
2017 \text { to 2027 (10 years) }\end{array}$ \\
\hline Forest & $56,609(39.8 \%)$ & $34,830(24.4 \%)$ & $25,798(18.2 \%)$ & $29,784(21.0 \%)$ & 31,556 & 5.95 \\
Grassland & $44,269(31.1 \%)$ & $46,186(32.3 \%)$ & $36,625(25.8 \%)$ & $31,550(22.2 \%)$ & $35,750.7$ & 13.31 \\
Shrub Land & $6418(4.5 \%)$ & $3477(2.4 \%)$ & $2738(1.9 \%)$ & $2495(1.8 \%)$ & 3065.4 & 22.86 \\
Cropland & $29,828(21.0 \%)$ & $56,415(39.5 \%)$ & $73,168(51.5 \%)$ & $75,574(53.2 \%)$ & $69,152.7$ & -8.50 \\
Bare Land & $5068(3.6 \%)$ & $1894(1.3 \%)$ & $3691(2.6 \%)$ & $2615(1.8 \%)$ & 2456.7 & -6.05 \\
Built up areas & $34(0.02 \%)$ & $44(0.03 \%)$ & $65(0.05 \%)$ & $80(0.06 \%)$ & 116.5 & 45.63 \\
\hline
\end{tabular}


Table 21. Land cover classes for 1987, 1997, 2007 and 2017 in Sand River sub-catchment.

\begin{tabular}{ccccc}
\hline Category & 1987 Area (Ha) & 1997 Area (Ha) & 2007 Area (Ha) & 2017 Area (Ha) \\
\hline Forest & $13,955(7.6 \%)$ & $9991(5.5 \%)$ & $8184(4.5 \%)$ & $7986(4.4 \%)$ \\
Grassland & $87,106(47.4 \%)$ & $86,467(47.2 \%)$ & $81,804(44.7 \%)$ & $79,884(43.6 \%)$ \\
Shrub Land & $80,217(43.6 \%)$ & $78,847(43.1 \%)$ & $76,801(42.0 \%)$ & $72,183(39.4 \%)$ \\
Bare Land & $2510(1.4 \%)$ & $7785(4.3 \%)$ & $16257(8.9 \%)$ & $22,977(12.6 \%)$ \\
\hline
\end{tabular}

Table 22. Land cover percentage changes by category per decade in Sand River sub-catchment.

\begin{tabular}{ccccc}
\hline Category & $\begin{array}{c}\text { 1987-1997 } \\
\text { \% Change }\end{array}$ & $\begin{array}{c}\text { 1997-2007 } \\
\text { \% Change }\end{array}$ & $\begin{array}{c}\text { 2007-2017 } \\
\text { \% Change }\end{array}$ & $\begin{array}{c}\text { 1987-2017 } \\
\text { Overall \% change }\end{array}$ \\
\hline Forest & -28.41 & -18.09 & -2.42 & -42.77 \\
Grassland & -0.73 & -5.39 & -2.35 & -8.29 \\
Shrub Land & -1.71 & -2.59 & -6.01 & -10.02 \\
Bare Land & 210.16 & 108.82 & 41.34 & 815.42 \\
\hline
\end{tabular}

Table 23. Projected land cover by 2027 in Sand River sub-catchment.

\begin{tabular}{|c|c|c|c|c|c|c|}
\hline Category & 1987 Area $(\mathrm{Ha})$ & $\begin{array}{l}1997 \text { Area } \\
(\mathrm{Ha})\end{array}$ & $\begin{array}{l}2007 \text { Area } \\
(\mathrm{Ha})\end{array}$ & $\begin{array}{l}2017 \text { Area } \\
\quad(\mathrm{Ha})\end{array}$ & $\begin{array}{l}\text { Projected land cover } \\
\text { by } 2027 \text { Area }(\mathrm{Ha})\end{array}$ & $\begin{array}{c}\% \text { change btw } 2017 \\
\text { to } 2027 \text { ( } 10 \mathrm{yrs})\end{array}$ \\
\hline Forest & $13,955(7.6 \%)$ & $9991(5.5 \%)$ & $8184(4.5 \%)$ & $7986(4.4 \%)$ & 8465.16 & 6 \\
\hline Grassland & $87,106(47.4 \%)$ & $86,467(47.2 \%)$ & $81,804(44.7 \%)$ & $79,884(43.6 \%)$ & $87,872.4$ & 10 \\
\hline Shrub Land & $80,217(43.6 \%)$ & $78,847(43.1 \%)$ & $76,801(42.0 \%)$ & $72,183(39.4 \%)$ & $76,513.98$ & 6 \\
\hline Bare Land & $2510(1.4 \%)$ & $7785(4.3 \%)$ & 16257 (8.9\%) & $22,977(12.6 \%)$ & $10,178.5$ & -55.70 \\
\hline
\end{tabular}

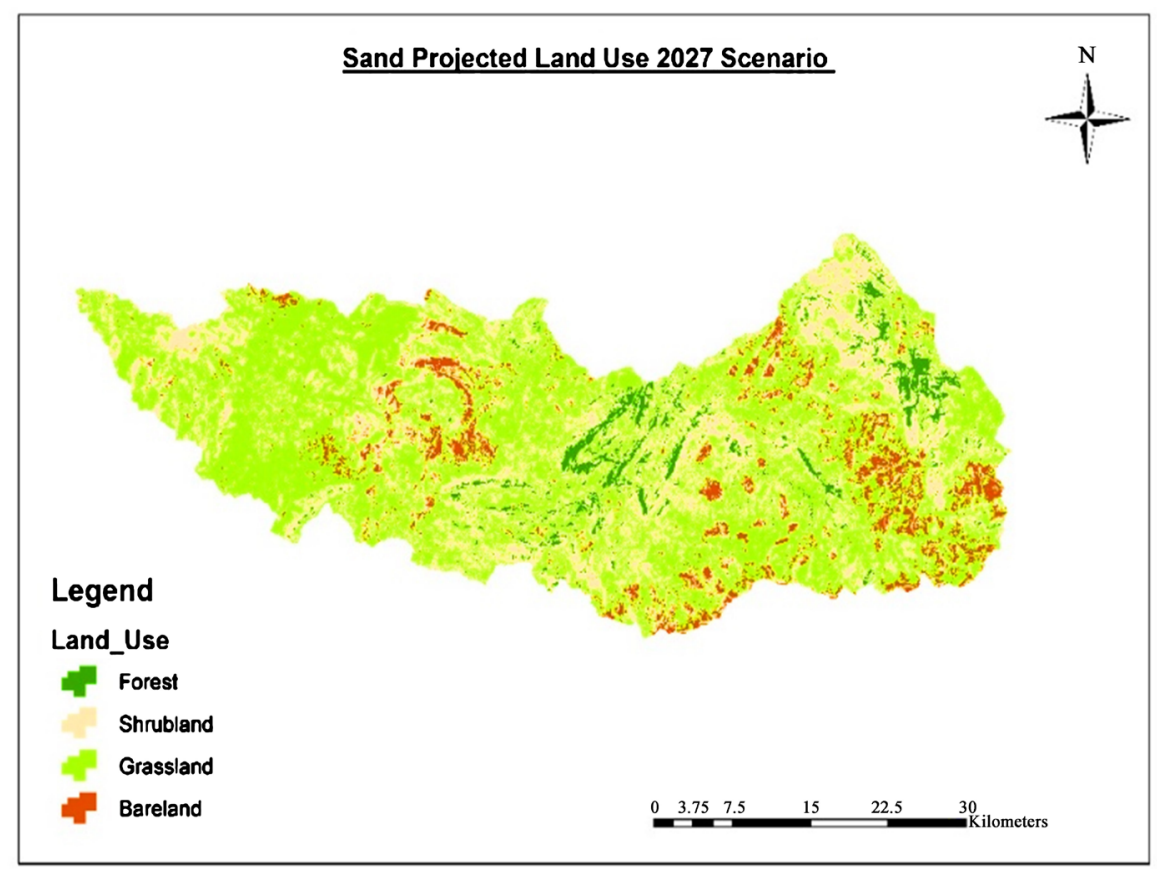

Figure 12. Map of predicted land cover change in Sand River sub-catchment by 2027. 


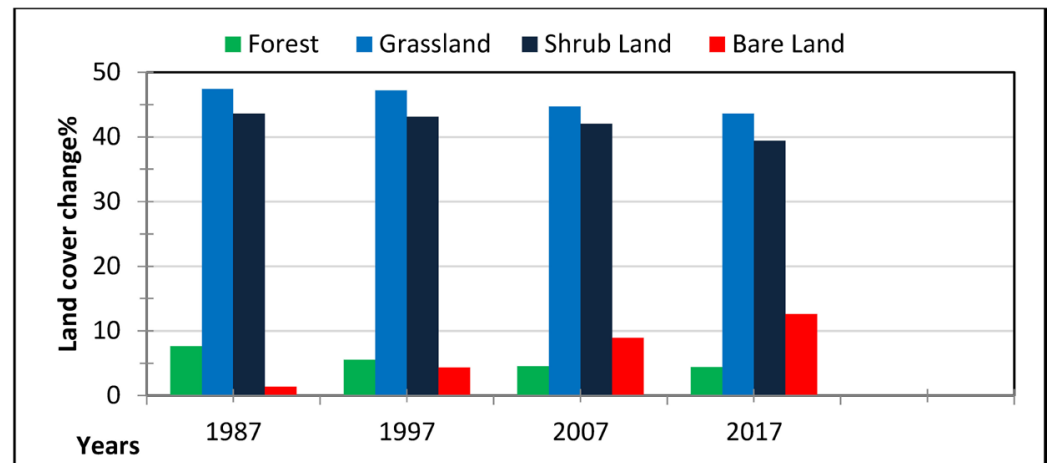

Figure 13. Percentage land cover change between 1987 and 2017 in the Sand River sub-catchment.



Figure 14. Projected land cover change by 2027 in the Sand River sub-catchment.

this resolution image to identify settlements and small crop lands.

\subsubsection{Projected Land Cover Change between 2017 and 2027 in Talek Sub-Catchment}

As was the case in the other three sub-catchments, Talek sub-catchment also exhibited some significant changes in land cover over the last 30 years (Tables 24-26). Precisely, between 1987 and 1997, bare land and grass lands decreased by $-55.04 \%$ and $-10.28 \%$, respectively, while forest land and shrub lands increased by $22.51 \%$ and $18.37 \%$, respectively. A slightly different trend was observed in the period between 1997-2007, whereby forest land and bare land decreased by $-49.31 \%$ and $-8.81 \%$, respectively, while shrub lands and grasslands increased by $7.04 \%$ and $2.43 \%$, respectively. A further decrease in forest land $(-35.63 \%)$ was observed in the 2007-2017 period, as did grass lands (-26.23\%). However, bare land and shrub lands increased by $34.06 \%$ and $22.93 \%$, respectively. Overall, shrub lands exhibited the largest change over the 30 -year period; increasing from $18.37 \%$ in 1987 to $56.28 \%$ in 2017. In addition, the greatest degradation of forest land, grass land and shrub lands occurred between 1987 and 2007 in the sub-catchment (Figures 15-17). As Talek sub-basin straddles part of Maasai Mara Game Reserves with scattered settlements, it was difficult for this resolution image to identify settlements and small crop lands. However, in 2017 
Table 24. Land cover classes for 1987, 1997, 2007 and 2017 in Talek sub-catchment.

\begin{tabular}{ccccc}
\hline Category & 1987 Area (Ha) & 1997 Area (Ha) & 2007 Area (Ha) & 2017 Area (Ha) \\
\hline Forest & $12,357(7.0 \%)$ & $15,138(8.6 \%)$ & $7673(4.4 \%)$ & $4939(2.8 \%)$ \\
Grassland & $92,493(52.8 \%)$ & $82,989(47.2 \%)$ & $85,005(48.4 \%)$ & $62,708(35.7 \%)$ \\
Shrub Land & $62,757(35.8 \%)$ & $74,286(42.2 \%)$ & $79,786(45.4 \%)$ & $98,079(55.9 \%)$ \\
Cropland & $0(0.0 \%)$ & $0(0.0 \%)$ & $0(0.0 \%)$ & $5607(3.2 \%)$ \\
Bare Land & $7723(4.4 \%)$ & $3472(2.0 \%)$ & $3166(1.8 \%)$ & $4244(2.4 \%)$ \\
\hline
\end{tabular}

Table 25. Trend and magnitude of land cover change \% (1987-2017) in the Talek sub-catchments.

\begin{tabular}{ccccc}
\hline Category & $\begin{array}{c}1987-1997 \\
\text { \% Change }\end{array}$ & $\begin{array}{c}\text { 1997-2007 } \\
\text { \% Change }\end{array}$ & $\begin{array}{c}\text { 2007-2017 } \\
\text { \% Change }\end{array}$ & $\begin{array}{c}\text { 1987-2017 } \\
\text { Overall \% change }\end{array}$ \\
Forest & 22.51 & -49.31 & -35.63 & -60.03 \\
Grassland & -10.28 & 2.43 & -26.23 & -32.20 \\
Shrub Land & 18.37 & 7.40 & 22.93 & 56.28 \\
Cropland & 0 & 0 & 0 & 100 \\
Bare Land & -55.04 & -8.81 & 34.06 & -45.04 \\
\hline
\end{tabular}

Table 26. Land cover 1987-2017 projected to 2027 in Talek sub-catchment.

\begin{tabular}{ccccccc}
\hline Category & 1987 Area (Ha) & 1997 Area (Ha) & 2007 Area (Ha) & 2017 Area (Ha) & $\begin{array}{c}\text { Projected land cover by } \\
\text { 2027 (Ha) }\end{array}$ & $\begin{array}{c}\text { \% change } \\
\mathbf{2 0 1 7 - 2 0 2 7}\end{array}$ \\
\hline Forest & $12,357(7.0 \%)$ & $15,138(8.6 \%)$ & $7673(4.4 \%)$ & $4939(2.8 \%)$ & 5136.56 & 4.0 \\
Grassland & $92,493(52.8 \%)$ & $82,989(47.2 \%)$ & $85,005(48.4 \%)$ & $62,708(35.7 \%)$ & $64,902.78$ & 3.5 \\
Shrub land & $62,757(35.8 \%)$ & $74,286(42.2 \%)$ & $79,786(45.4 \%)$ & $98,079(55.9 \%)$ & $100,040.58$ & 2.0 \\
Cropland & $0(0.0 \%)$ & $0(0.0 \%)$ & $0(0.0 \%)$ & $5607(3.2 \%)$ & 3364.2 & -40.0 \\
Bare land & $7723(4.4 \%)$ & $3472(2.0 \%)$ & $3166(1.8 \%)$ & $4244(2.4 \%)$ & 2546.676 & -40.0 \\
\hline
\end{tabular}

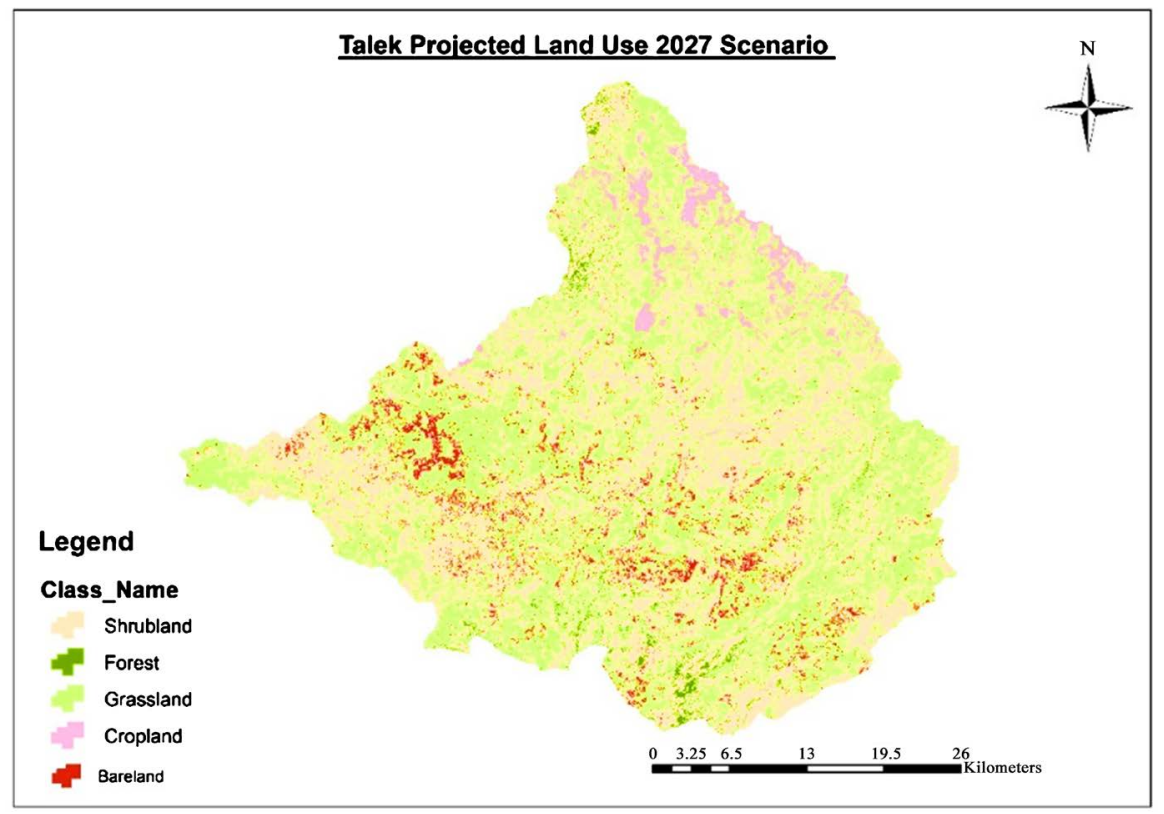

Figure 15. Map of predicted land cover change by 2027 in Talek sub-catchment. 


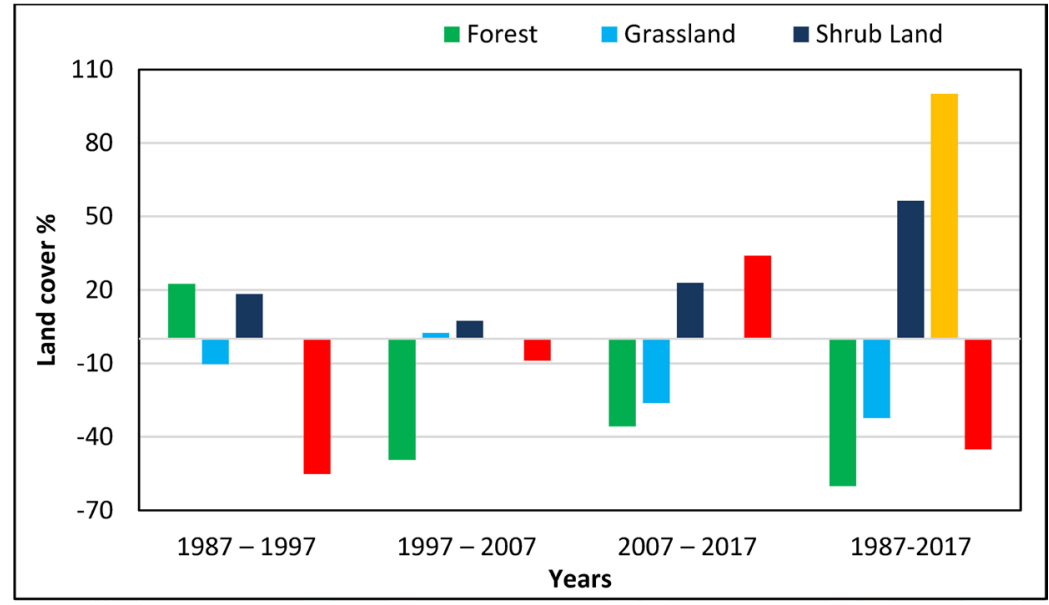

Figure 16. Decadal land cover change percentage in Talek sub-catchment.

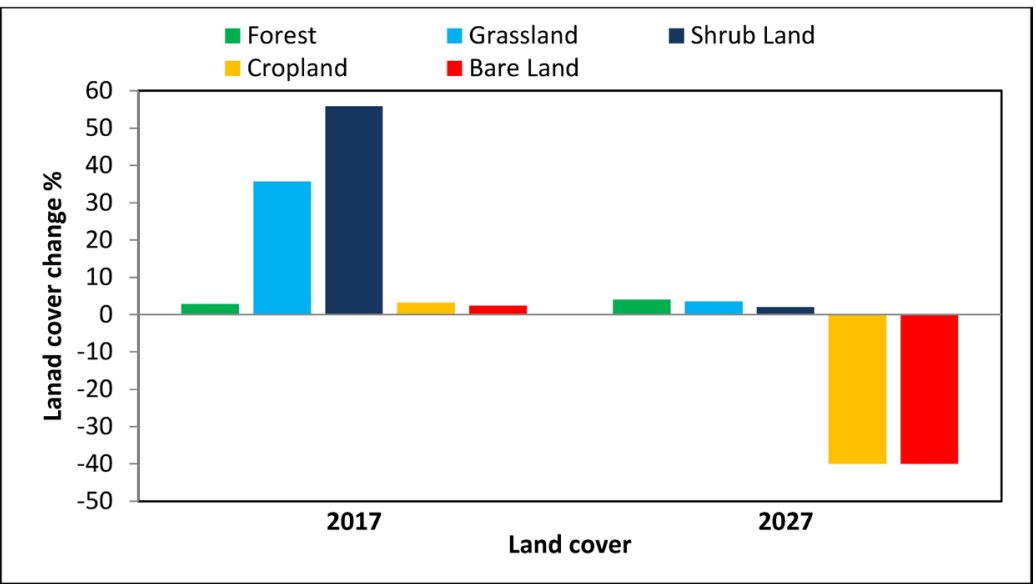

Figure 17. Projected land cover change by 2027 in Talek sub-catchment.

a slight increase in crop land of 5607 ha (3.2\%) was observed in Talek sub-catchment.

\section{Discussion}

Land cover change in the four sub-basins of the Mara River basin i.e. Amala, Nyangores, Sand River and Talek was evident though variable and apparently nonlinear. The dynamics of land cover change were however rather gradual, implying that different development trends in each decadal period depended on human activities and climatic factors; and area specific interventions. In fact, studies show that the pace, magnitude and spatial reach of human alterations of the Earth's land surface are unprecedented; with changes in land cover being among the most important [33]. In the current study, spatial analysis revealed that the observed land cover changes were distributed across the Mara River basin sub-catchments and included multiple change directions in LC. Similar land cover change trends have been reported in previous studies in other regions [34].

High rate of deforestation averaging 5.3\% per annum was observed within the Mara River basin, but was more pronounced in the two upper Mara River sub-catchments (Amala and Nyangores). In addition, nonlinearity of land cover 
changes in the last 30 years was also illustrated by some distinctive categories of LC in the present study. For instance, the built-up areas in the study area increased significantly between 2007 and 2017. A number of forces such as continuous logging and cropping, human settlements triggered by the ever growing human population as well as changes in climatic factors could be driving the observed changes in land cover within the basin. Thus, it is important to track the dynamic of LC change in these areas, and understand the underlying driving forces of these changes. This would help in predicting future pattern of LC change in the region to inform policy.

Rapid population growth and changes in climatic conditions have been singled out as some of the major drivers of deforestation in attempts to increase agricultural land and human settlements in the Mara River basin. Despite improvements in land-cover characterization made possible by earth observing satellites, global and regional land covers are still poorly enumerated [35]. However, scientists recognize that the magnitude of land cover change is massive. One earlier estimate, for example, holds that the global expansion of croplands since 1850 has converted some 6 million $\mathrm{km}^{2}$ of forests/woodlands and 4.7 million $\mathrm{km}^{2}$ of savannas/grasslands/steppes, while 0.6 million $\mathrm{km}^{2}$ of cropland has been abandoned [36].

In the current study, the period between 1987 and 1997, exhibited a much higher reduction in spatial expansion in land cover, particularly in forest land compared to the period between 2007 and 2017. This could be attributed to a number of reasons, key among them laxity in enforcement of law on logging and encroachment of the Mau forests. However, from 2007 onwards, the basin witnessed a steady growth in forest cover after the Kenyan government's spirited intervention to reclaim most parts of the Mau forest previously occupied by communities. Previous studies show that reclamation practices create a diverse and valuable forest of native trees that produce a number of benefits to the ecosystem and to the inhabitants [37]. In all the four sub-catchments, a significant difference in forest cover change was observed though to varying degrees. A similar trend was also observed in crop lands, grasslands and bare land.

Temperature and precipitation were identified as significant actors in the observed changes in land cover within the Mara River basin. When the mean total annual precipitation was high and maximum mean annual temperature was low, the NDVI of all land cover categories recorded highest values, whereas the opposite was true. Consistent with the findings of the present study, Herrmann et al. [38] reported a close relationship between rainfall and surface greenness in the Sahel region on a large scale, while variations in other climatic and environmental factors were considered of minimal effect. On the contrary, some researchers argue that the relationship between rainfall and vegetation greenness can be explained by changes in soil moisture conditions, which cause an instantaneous plant response [39]. Although variation of soil moisture in semiarid and arid regions is significantly controlled by rainfall amount, near-surface air tem- 
perature may also be considered an additional climatic factor in soil moisture changes [40] [41] [42]. Therefore, the effect of air temperature on the relationship between rainfall and vegetation greenness should be examined in greater detail for tropical ecosystems, which most previous studies have not taken into account.

Following the correlation observed between different land cover categories and climatic factors (annual mean precipitation and maximum temperature) in the four Mara River sub-catchments in the context of different anthropogenic activities, chances that loss in land cover can be restored is eminent. Indeed, going by the predictions emanating from this study, there is a likelihood of improvement in land cover in the Mara River basin sub-catchments by the year 2027. This will however depend on continued protection of the Mara River basin by the inhabitants themselves, Non-governmental Organizations like Wild Wide Funds for Nature (WWF), County governments of Nakuru, Narok and Bomet as well as assistance from the national government. It is therefore critical to encourage the aforesaid communities and partners to protect and conserve their environment for long term benefits. Indeed, between the years 1987 and 1997, there was a reduction in forest cover within the Mara River sub-catchment compared to the period 2007-2017 period. There is a possibility of similar reduction in vegetation cover over the next 27 years if there are lapses in the implementation and enforcement of environmental laws and regulations.

After the initial reduction in forest cover and a corresponding increase in bare land and built-up areas between 1987 and 2007, the Mara River sub-catchments witnessed a steady expansion of various land cover classes. The trend may indeed continue even after 2027 and beyond as suggested by the land cover change projections in the present study. Nevertheless, for these projections to be realized, it is suggested here that deliberate attempts must be made by the government of Kenya and the local communities to deliberately protect the environment. This will in turn lead to improved economy for the community and the government as well as improve the overall health of the Mara River ecosystem.

\section{Conclusion}

This study highlights the impact of changing climatic factors on different land cover categories within the four Mara River basin sub-catchments. Generally, land cover categories exhibited positive sensitivity to high precipitation and low temperature. On the contrary, high temperature exhibited strong negative correlations with the different land cover categories including croplands. The results however suggest that, with proper interventions, forest land, grassland, shrub land and even built-up areas are likely to increase, while crop land and bare lands are likely to decrease by 2027 .

\section{Acknowledgements}

The authors acknowledge the help and assistance received from community 
members and local leaders within the Mara River basin. We are also grateful to all the enumerators who helped in the administration of questionnaires in the study area.

\section{Funding}

This research received no external funding.

\section{Conflicts of Interest}

The authors declare no conflicts of interest regarding the publication of this paper.

\section{References}

[1] Li, J., Zhang, Y., Qin, Q. and Yan, Y. (2017) Investigating the Impact of Human Activity on Land Use/Cover Change in China's Lijiang River Basin from the Perspective of Flow and Type of Population. Sustainability, 9, 383.

https://doi.org/10.3390/su9030383

[2] Pletterbauer, F., Melcher, A., Graf, W. (2018) Climate Change Impacts in Riverine Ecosystems. In: Schmutz, S. and Sendzimir, J., Eds., Riverine Ecosystem Management, Aquatic Ecology Series, Springer, Cham, 203-223.

https://doi.org/10.1007/978-3-319-73250-3_11

[3] Mango, L.M., Melesse, A.M., Mcclain, M.E., Gann, D. and Setegn, S.G. (2011) Land Use and Climate Change Impacts on the Hydrology of the Upper Mara River Basin, Kenya: Results of a Modeling Study to Support Better Resource Management. $\mathrm{Hy}$ drology and Earth System Sciences, 15, 2245-2258. https://doi.org/10.5194/hess-15-2245-2011

[4] FAO (2011) The State of the World's Land and Water Resources for Food and Agriculture (SOLAW)-Managing Systems at Risk. FAO and Earthscan, Rome and London, $308 \mathrm{p}$.

[5] Ye, Y.Q., Zhang, J.E., Bryan, B.A., Gao, L., Qin, Z., Chen, L.L. and Yang, J.Y. (2018) Impacts of Rapid Urbanization on Ecosystem Services along Urban-Rural Gradients: A Case Study of the Guangzhou-Foshan Metropolitan Area, South China. Écoscience, 25, 235-247. https://doi.org/10.1080/11956860.2018.1442086

[6] Myhre, G., Shindell, D., Bréon, F. M., Collins, W., Fuglestvedt, J., Huang, J., et al. (2013) Anthropogenic and Natural Radiative Forcing. In: Stocker, T.F., Qin, D., Plattner, G.K., Tignor, M., Allen, S.K., Doschung, J., Nauels, A., Xia, Y., Bex, V. and Midgley, P.M., Eds., Climate Change 2013: The Physical Science Basis. Contribution of Working Group I to the 5 th Assessment Report of the IPCC, Cambridge University Press, Cambridge, 659-740.

[7] Liu, Y., Li, Y., Li, S. and Motesharrei, S. (2015) Spatial and Temporal Patterns of Global NDVI Trends: Correlations with Climate and Human Factors. Remote Sensing, 7, 13233-13250. https://doi.org/10.3390/rs71013233

[8] Guo, H., Qi, H. and Jiang, T. (2008) Annual and Seasonal Streamflow Responses to Climate and Land-Cover Changes in the Poyang Lake Basin. Journal of Hydrology, 355, 106-122. https://doi.org/10.1016/j.jhydrol.2008.03.020

[9] Ichii, K., Kawabata, A. and Yamaguchi, Y. (2002) Global Correlation Analysis for NDVI and Climate Variables and NDVI Trends: 1982-1990. International Journal of Remote Sensing, 23, 3873-3878. https://doi.org/10.1080/01431160110119416 
[10] Luo, L., Wang, Z.M., Song, K.S., Zhang, B., Liu, D.W., Ren, C.Y. and Zhang, S.M. (2009) Research on the Correlation between NDVI and Climatic Factors of Different Vegetation in the Northeast China. Acta Botanica Boreali-Occidentalia Sinica, 29, 800-808.

[11] Zhang, Y.D., Zhang, X.H. and Liu, S.R. (2011) Correlation Analysis on Normalized Difference Vegetation Index (NDVI) of Different Vegetation and Climatic Factors in Southwest China. Chinese Journal of Applied Ecology, 22, 323-330.

[12] Chuai, X.W., Huang, X.J., Wang, W.J. and Bao, G. (2013) NDVI, Temperature and Precipitation Changes and Their Relationships with Different Vegetation Types during 1998-2007 in Inner Mongolia, China. International Journal of Climatology, 33, 1696-1706. https://doi.org/10.1002/joc.3543

[13] Qiu, G.Y., Yin, J., Tian, F. and Geng, S. (2011) Effects of the "Conversion of Cropland to Forest and Grassland Program" on the Water Budget of the Jinghe River Catchment in China. Journal of Environmental Quality, 40, 1745-1755.

https://doi.org/10.2134/jeq2010.0263

[14] Pang, G.J., Wang, X.J. and Yang, M.X. (2017) Using the NDVI to Identify Variations in, and Responses of, Vegetation to Climate Change on the Tibetan Plateau from 1982 to 2012. Quaternary International, 444, 87-96.

https://doi.org/10.1016/j.quaint.2016.08.038

[15] Roerink, G.J., Menenti, M., Soepboer, W. and Su, Z. (2003) Assessment of Climate Impact on Vegetation Dynamics by Using Remote Sensing. Physics and Chemistry of the Earth, 28, 103-109. https://doi.org/10.1016/S1474-7065(03)00011-1

[16] Meng, M., Ni, J. and Zong, M.J. (2011) Impacts of Changes in Climate Variability on Regional Vegetation in China: NDVI-Based Analysis from 1982 to 2000. Ecological Research, 26, 421-428. https://doi.org/10.1007/s11284-011-0801-z

[17] Crucifix, M., Betts, R.A. and Cox, P.M. (2005) Vegetation and Climate Variability: A GCM Modelling Study. Climate Dynamics, 24, 457-467. https://doi.org/10.1007/s00382-004-0504-Z

[18] Muhati, G.L., Olago, D. and Olaka, L. (2018) Past and Projected Rainfall and Temperature Trends in a Sub-Humid Montane Forest in Northern Kenya Based on the CMIP5 Model Ensemble. Glob. Global Ecology and Conservation, 16, e00469. https://doi.org/10.1016/j.gecco.2018.e00469

[19] Guan, D.J., Li, H.F., Inohae, T., Su, W.C., Nagaie, T. and Hokao, K. (2011) Modeling Urban Land Use Change by the Integration of Cellular Automaton and Markov Model. Ecological Modelling, 222, 3761-3772.

https://doi.org/10.1016/j.ecolmodel.2011.09.009

[20] Lu, Y.T., Wu, P.H., Ma, X.S. and Li, X.H. (2019) Detection and Prediction of Land Use/Land Cover Change Using Spatiotemporal Data Fusion and the Cellular Automata-Markov Model. Environmental Monitoring and Assessment, 191, Article No. 68. https://doi.org/10.1007/s10661-019-7200-2

[21] Menon, S., Rose, J., Khan, M.L. and Bawa, K.S. (2001) Identifying a Land-Use in the Conservation-Priority Areas Tropics: Change Modeling Approach. Conservation Biology, 15, 501-512. https://doi.org/10.1046/j.1523-1739.2001.015002501.x

[22] Wickramasuriya, R.C., Bregt, A.K., Van Delden, H. and Hagen-Zanker, A. (2009) The Dynamics of Shifting Cultivation Captured in an Extended Constrained Cellular Automata Land Use Model. Ecological Modelling, 220, 2302-2309. https://doi.org/10.1016/j.ecolmodel.2009.05.021

[23] Freier, K.P., Schneider, U.A. and Finckh, M. (2011) Dynamic Interactions between Vegetation and Land Use in Semi-Arid Morocco: Using a Markov Process for Mod- 
eling Rangelands under Climate Change. Working Paper. Agriculture, Ecosystems \& Environment, 140, 462-472. https://doi.org/10.1016/j.agee.2011.01.011

[24] Agarwal, C., Green, G.M., Grove, J.M., Evans, T.P. and Schweik, C.M. (2002) A Review and Assessment of Land-Use Change Models: Dynamics of Space, Time, and Human Choice. GTR NE-297. U.S. Department of Agriculture, Forest Service, Northeastern Research Station, Newton Square, PA, 61 p. https://doi.org/10.2737/NE-GTR-297

[25] Hamad, R., Balzter, H. and Kolo, K. (2018) Predicting Land Use/Land Cover Changes Using a CA-Markov Model under Two Different Scenarios. Sustainability, 10, 3421. https://doi.org/10.3390/su10103421

[26] Weng, Q. (2002) Land Use Change Analysis in the Zhujiang Delta of China Using Satellite Remote Sensing, GIS and Stochastic Modelling. Journal of Environmental Management, 64, 273-284. https://doi.org/10.1006/jema.2001.0509

[27] Sinha, P. and Kumar, L. (2013) Independent Two-Step Thresholding of Binary Images in Inter-Annual Land Cover Change/No-Change Identification. ISPRS Journal of Photogrammetry and Remote Sensing, 81, 31-43. https://doi.org/10.1016/j.isprsjprs.2013.03.010

[28] Agarwal, C., Green, G.M., Grove, J.M., Evans, T.P. and Schweik, C.M. (2002) A Review and Assessment of Land-Use Change Models: Dynamics of Space, Time and Human Choice. USDA Forest Service, Gen. Tech. Rep. NE-297. https://www.nrs.fs.fed.us/pubs/gtr/gtr_ne297.pdf

[29] Eastman, J.R. (2009) Idrisi Taiga, Guide to GIS and Image Processing, Manual Version 16.02. Clark University, Worcester, $342 \mathrm{p}$.

[30] Pontius. R.G. and Malanson, J. (2005) Comparison of the Structure and Accuracy of Two Land Change Models. International Journal of Geographical Information Science, 19, 243-265. https://doi.org/10.1080/13658810410001713434

[31] Dida, O.G., Anyona, D.N., Abuom, P.O., Akoko, D., Adoka, S. O., Matano, A.S., Owuor, P.O. and Ouma, C. (2018) Spatial Distribution and Habitat Characterization of Mosquito Species during the Dry Season Along the Mara River and Its Tributaries, in Kenya and Tanzania. Infectious Diseases of Poverty, 7, Article No. 2. https://doi.org/10.1186/s40249-017-0385-0

[32] Vázquez-Jiménez, R., Romero-Calcerrada, R., Novillo, C.J., Ramos-Bernal, R.N. and Arrogante Funes, P. (2017) Applying the Chi-Square Transformation and Automatic Secant Thresholding to Landsat Imagery as Unsupervised Change Detection Methods. Journal of Applied Remote Sensing, 11, Article ID: 016016. https://doi.org/10.1117/1.JRS.11.016016

[33] Geist, H.J. and Lambin, E.F. (2002) Proximate Causes and Underlying Driving Forces of Tropical Deforestation. Bioscience, 52, 143-150. https://doi.org/10.1641/0006-3568(2002)052[0143:PCAUDF]2.0.CO;2

[34] Pagnutti, C., Bauch, C.T. and Anand, M. (2013) Outlook on a Worldwide Forest Transition. PLoS ONE, 8, e75890. https://doi.org/10.1371/journal.pone.0075890

[35] Intergovernmental Panel on Climate Change (2000) Land Use, Land-Use Change, and Forestry. Special Report, Cambridge Univ. Press, Cambridge.

[36] Ramankutty, N. and Foley, J.A., (1999) Estimating Historical Changes in Global Land Cover: Croplands from 1700 to 1992. Global Biogeochemical Cycles, 13, 997-1027. https://doi.org/10.1029/1999GB900046

[37] Zipper, C.E., Burger, J.A., Skousen, J.G., Angel, P.N., Barton, C.D., Davis, V. and Franklin, J.A. (2011) Restoring Forests and Associated Ecosystem Services on Ap- 
palachian Coal Surface Mines. Environment Management, 47, 751-765.

https://doi.org/10.1007/s00267-011-9670-Z

[38] Herrmann, S.M., Anyamba, A. and Tucker, C.J. (2005) Recent Trends in Vegetation Dynamics in the African Sahel and Their Relationship to Climate. Global Environmental Change, 15, 394-404. https://doi.org/10.1016/j.gloenvcha.2005.08.004

[39] Tracol, Y., Mougin, E., Hiernaux, P. and Jarlan, L. (2006) Testing a Sahelian Grassland Functioning Model Against Herbage Mass Measurements. Ecological Modelling, 193, 437-446. https://doi.org/10.1016/j.ecolmodel.2005.08.033

[40] Cho, J., Komatsu, H., Pokhrel, Y., Yeh, P.J.F., Oki, T. and Kanae, S. (2011) the Effects of Annual Precipitation and Mean Air Temperature on Annual Runoff in Global Forest Regions. Climatic Change, 108, 401-410.

https://doi.org/10.1007/s10584-011-0197-3

[41] Gonzalez, P., Tucker, C.J. and Sy, H. (2012) Tree Density and Species Decline in the African Sahel Attributable to Climate. Journal of Arid Environments, 78, 55-64. https://doi.org/10.1016/j.jaridenv.2011.11.001

[42] Komatsu, H., Cho, J., Matsumoto, K. and Otsuki, K. (2012) Simple Modeling of the Global Variation in Annual Forest Evapotranspiration. Journal of Hydrology, 420-421, 380-390. https://doi.org/10.1016/j.jhydrol.2011.12.030 